\title{
Controller Design for a Second-Order Plant with Uncertain Parameters and Disturbance: Application to a DC Motor
}

\author{
Alejandro Rincón, ${ }^{1}$ Fredy E. Hoyos, ${ }^{2}$ and Fabiola Angulo ${ }^{2}$ \\ ${ }^{1}$ Facultad de Ingeniería y Arquitectura, Universidad Católica de Manizales, Cr 23 No 60-63, Manizales 170002, Colombia \\ ${ }^{2}$ Departamento de Ingeniería Eléctrica, Electrónica y Computación, Facultad de Ingeniería y Arquitectura, \\ Universidad Nacional de Colombia, Sede Manizales, Percepción y Control Inteligente, Bloque Q, Campus La Nubia, \\ Manizales 170003, Colombia
}

Correspondence should be addressed to Fabiola Angulo; fangulog@unal.edu.co

Received 1 August 2012; Revised 20 December 2012; Accepted 28 December 2012

Academic Editor: Gani Stamov

Copyright ( $) 2013$ Alejandro Rincón et al. This is an open access article distributed under the Creative Commons Attribution License, which permits unrestricted use, distribution, and reproduction in any medium, provided the original work is properly cited.

\begin{abstract}
This paper shows the controller design for a second-order plant with unknown varying behavior in the parameters and in the disturbance. The state adaptive backstepping technique is used as control framework, but important modifications are introduced. The controller design achieves mainly the following two benefits: upper or lower bounds of the time-varying parameters of the model are not required, and the formulation of the control and update laws and stability analysis are simpler than closely related works that use the Nussbaum gain method. The controller has been developed and tested for a DC motor speed control and it has been implemented in a Rapid Control Prototyping system based on Digital Signal Processing for dSPACE platform. The motor speed converges to a predefined desired output signal.
\end{abstract}

\section{Introduction}

An important challenge for controller design plants is the unknown time-varying behavior of its parameters (cf [1-3]). The state adaptive backstepping (SAB) of [4] is an important framework to design this kind of controllers (see [5-9]). In the adaptive controllers that are based on the SAB and do not use the Nussbaum gain method, the transient behavior of the tracking error is upper bounded by an unknown positive constant, as can be noticed from [5,10-13]. Such constant bound is the function of (i) constant upper bounds of varying bounded plant model parameters, (ii) constant plant model parameters, (iii) user-defined parameters of the update laws, and (iv) the initial values of the plant model states. In addition, it does not involve integral terms that depend on Nussbaum functions. Therefore, the constant upper bound of the tracking error can be made small by choosing large values of the update law gains. This would ensure that the tracking error takes on small values. To handle the effect of unknown varying behavior of plant model parameters, the robustness and Nussbaum gain techniques are usually combined with $\mathrm{SAB}$ control schemes.
The robust-SAB control schemes involve a control law with a compensation term and a modification of the update law, for example, the projection type modification (see [14, $15]$ ), or the $\sigma$ modification (see [16-19]). The main drawback of this technique is the following:

(D1) upper or lower bounds of the plant coefficients are required to be known to achieve the asymptotic convergence of the tracking error to a residual set of user-defined size.

On the other hand, neural networks allow to represent part of the nonlinear behavior of real systems and can take into account the time-varying behavior. In the case of completely unknown systems, they represent the whole plant model terms. Usually, the use of neural networks leads to approximation error, which is nonlinear and possibly time varying. The effect of this term has been tackled by means of robust adaptive control schemes based on the Lyapunov or the Lyapunov-like function; see $[15,19]$. Those adaptive controllers exhibit some drawbacks, as shown in the following. In [19], a nonlinear system in control affine strict-feedback form is considered, and a neural network SAB control scheme 
is designed. The unknown state-dependent terms are represented by RBF neural networks, with unknown coefficients and known basis functions. The following assumptions are made: (i) the mentioned coefficients are unknown and upper bounded by known positive constants, and (ii) the identification error is upper bounded by an unknown constant. The update laws allow to tackle the effect of the unknown coefficients of the RBF representation. In that paper, the stability analysis indicates that the Lyapunov function converges to a residual set whose size depends on both the upper bound of the identification error and the upper bound of the coefficients of the RBF representation. Therefore, the tracking error converges to a residual set whose size depends on those bounds. Hence, those upper bounds must be known to obtain the convergence of the tracking error to a residual set of user-defined size. In [15] a nonlinear system in control affine state space form is considered, and a neural network based on output adaptive backstepping (OAB) is considered. The unknown nonlinear state-dependent terms are represented by neural networks with unknown coefficients and known basis functions. The following assumptions are made: (i) the coefficients of representation are unknown and constant, and (ii) the identification error term is upper bounded by a constant. The projection type update laws allow to tackle the effect of the unknown coefficients of representation. In that paper, the stability analysis indicates that the Lyapunov function converges to a residual set whose size depends on the upper bound of the identification error term. Therefore, the tracking error converges to a residual set whose size also depends on the identification error. Hence, the main drawback of the mentioned adaptive control schemes is the following:

(D2) the upper bound of the identification error term must be known to achieve the convergence of the tracking error to a residual set of user-defined size.

As can be noticed from [20-24], Nussbaum-SAB control schemes are usually based on the schemes in [25-27] which are in turn based on the Universal Stabilizer that was originally presented in [28] and discussed in [29, pages 335$338]$. As can be concluded from [20, 21], a proper design of the Nussbaum-SAB control scheme overcomes the main drawback of the mentioned $\sigma$ and projection based robust$\mathrm{SAB}$ control schemes, as upper or lower bounds of the plant model parameters are not required to be known, and the convergence of the tracking error to a residual set of user-defined size is guaranteed. Other recent Nussbaum-SAB control schemes indicate that the Nussbaum gain technique exhibits the following drawback:

(D3) the upper bound of the transient behavior of the Lyapunov function depends on integral terms that involve Nussbaum functions and have the time as the upper limit of the integral operation (see [24, page 477], [20, page 1791], [6, page 856], and [30, page 4639]). Therefore, the upper bound of the transient behavior of the tracking error depends on such integral terms, so that the tracking error may take on overly large values.
This is in agreement with the violent behavior mentioned in [29, page 337]. In addition, some of the control schemes that use this technique have the following drawbacks: (i) some upper or lower bounds of the plant coefficients are required to be known in order to guarantee the asymptotic convergence of the tracking error to a residual set of user-defined size as in $[25,30]$, and (ii) the control or update laws involve signum type signals as in $[24,31]$.

SAB control schemes have been developed and applied to motors, and some of them include the incorporation of the Nussbaum and robustness techniques. In [5] an adaptive controller is designed for a linear motor drive. The mathematical model used to describe the motor is in controllable form, and the friction coefficients are assumed constant and unknown. Nevertheless, the upper bound of the disturbance term and the upper and lower bounds of the friction coefficients are required to be known. In [6] an adaptive controller based on the Nussbaum gain technique and the $\sigma$ modification of the update law is designed for a class of SISO systems and applied to a DC motor turning a robotic load. Each differential equation of the SISO system involves an additive and unknown disturbance-like term, which is upper bounded by a known nonnegative function with unknown coefficients. Nevertheless, the upper and lower bounds of the plant model parameters must be known to guarantee the convergence of the tracking error to a residual set of user-defined size, and the upper bound of the transient behavior of the tracking error depends on integral terms that involve the Nussbaum functions. The last drawback is common in Nussbaum adaptive control schemes. In [7], an adaptive controller is designed for the position control of an $x-y-\Theta$ motion control stage using a linear ultrasonic motor. The friction force includes the static friction, Coulomb friction, and viscous friction. The idea is to control the $x$-axis, $y$-axis, and $\Theta$-axis, separately. A lumped uncertainty term results from the unknown parameter variations and external force disturbances. The lumped uncertainty is represented by means of an adaptive fuzzy neural network. The identification error is defined as the difference between the real value of the lumped parameter and the representation based on the Sugeno adaptive fuzzy neural network. Such identification error is handled by means of an updated parameter provided by an additional update law and an input compensator. The Lyapunov function includes a quadratic form depending on the difference between the identification error and its updated value. Nevertheless, the time derivative of the Lyapunov function neglects the time derivative of the identification error; see page 681. It amounts to assume that the identification error is constant or zero in the Lyapunov function. In [8], a linear induction motor is considered and the goal is to control the mover position. The friction force and the unknown time varying model parameters are lumped into an unknown term whose upper bound is constant and unknown. The lumped unknown term is represented by a radial basis function network (RBFN), estimated in real time. The reconstructed error is defined as the difference between the lumped term and the representation based on RBFN. 
The effect of the reconstructed error is tackled by means of an updated parameter provided by an additional updating law. The Lyapunov function involves a quadratic form for the difference between the reconstructed error and its updated parameter. The drawback is that the time derivative of the reconstructed error is neglected in the time derivative of the Lyapunov function, which is not realistic and could degrade the robustness of the controller. In summary, the main drawbacks of the above control schemes are the following:

(D4) upper and lower bounds of plant model parameters and lumped plant model terms are required to be known;

(D5) the upper bound of the transient behavior of the tracking error depends on integral terms that involve Nussbaum functions;

(D6) the time derivative of identification error is neglected in the time derivative of the Lyapunov function.

In the present work, an adaptive controller is developed for a permanent magnet DC motor. The state adaptive backstepping ( $\mathrm{SAB}$ ) of [4] is used as the basic framework for the controller design. In order to handle the unknown varying model parameters, significant modifications are introduced in the approach, on the basis of the modifications appearing in [32]. The main modifications are as follows: (i) use a truncated version of the quadratic form that depends on the backstepping states, and (ii) develop a convergence analysis based on the truncated version of the quadratic form. Using the scheme proposed in this paper, the following benefits are obtained:

(RC1) the resulting upper bound of the transient behavior of the tracking error is constant and does not depend on integral terms involving Nussbaum functions, so that the transient behavior of the tracking error can be rendered small by properly choosing the controller parameters;

(RC2) none of the exact values of the plant model parameters are required to be known;

(RC3) none of the upper bounds of the plant model parameters are required to be known;

(RC4) the tracking error converges to a residual set whose size is user defined, despite the lack of knowledge on both the exact values and the upper bounds of the plant model parameters;

(RC5) discontinuous signals are avoided in the control and update laws;

(RC6) the time derivative of the Lyapunov function does not neglect the time derivative of any varying parameter.
The controller was applied to a permanent magnet DC motor whose voltage input is supplied by a buck power converter. With the aim to obtain a good agreement between simulations and experimental set-up, the numerical simulation includes realistic characteristics such as internal resistances, discretization, and time delay. The controller was implemented in a digital platform. The control design procedure and the stability analysis indicate that the drawbacks (D1), (D2), (D3), (D4), (D5), and (D6) are overcome, as the benefits (RC1) to (RC6) of the control scheme in [32] are achieved in the present work. In addition, the bounded nature of all the closed loop signals is guaranteed.

This paper is organized as follows. In Section 2 the plant model used to design the controller and the goal of the control are presented. In Section 3 the design of the controller is developed. In Section 4 the bounded nature of the closed loop signals and the convergence of the tracking error are proven. In Section 5 numerical and experimental results are presented, and finally, Section 6 is devoted to conclusions.

\section{Plant Model and Control Goal}

The linear model corresponding to a DC permanent magnet motor is given by

$$
\begin{gathered}
\dot{W}_{m}=-\frac{B}{J_{e q}} W_{m}+\frac{k_{t}}{J_{e q}} i_{a}-\frac{\left(T_{\text {fric }}+T_{L}\right)}{J_{e q}}, \\
\dot{i}_{a}=-\frac{R_{a}}{L_{a}} i_{a}-\frac{k_{e}}{L_{a}} W_{m}+\frac{1}{L_{a}} u .
\end{gathered}
$$

The state variables are the armature current $i_{a}$ and the motor speed $W_{m}$. The control input is $u=v_{c}$ (i.e., the capacitor voltage supplied by the buck converter) and the output of the system is $y=W_{m} . k_{e} ;[\mathrm{V} / \mathrm{rad} / \mathrm{s}]$ is the voltage constant, $L_{a}[\mathrm{mH}]$ is the armature inductance, $R_{a}[\Omega]$ is the armature resistance, $B[\mathrm{~N} \cdot \mathrm{m} / \mathrm{rad} / \mathrm{s}]$ is the viscous friction coefficient, $J_{e q}$ $\left[\mathrm{kg} \cdot \mathrm{m}^{2}\right]$ is the inertia moment, $k_{t}[\mathrm{~N} \cdot \mathrm{m} / \mathrm{A}]$ is the motor torque constant, $T_{\text {fric }}[\mathrm{N} \cdot \mathrm{m}]$ is the friction torque, and $T_{L}[\mathrm{~N} \cdot \mathrm{m}]$ is the load torque.

Remark 1. The only objective of the buck converter is to supply the voltage value obtained from the controller law. For this reason it is not taken into account in the controller design.

The following assumptions for the model (1) are made: (Ai) the parameters $T_{L}$ and $J_{e q}$ vary with time but they are upper and lower bounded by unknown constants, (Aii) the parameters $B, k_{t}, R_{a}, L_{a}, k_{e}$ are unknown and constant, and (Aiii) $W_{m}$ and $i_{a}$ are measured. The plant model (1) can be rewritten as

$$
\begin{gathered}
\dot{x}_{1}=-a_{1} x_{1}+a_{2} x_{2}-a_{3}, \\
\dot{x}_{2}=-a_{4} x_{1}-a_{5} x_{2}+b u, \\
x_{1}=W_{m}, \quad x_{2}=i_{a}, \quad u=v_{c}, \quad y=W_{m},
\end{gathered}
$$


where $a_{1}=B / J_{e q}, a_{2}=k_{t} / J_{e q}, a_{3}=\left(T_{\text {fric }}+T_{L}\right) / J_{e q}, a_{4}=$ $k_{e} / L_{a}, a_{5}=R_{a} / L_{a}$, and $b=1 / L_{a}$ are positive constants. Assumption (Ai) implies that parameters $a_{1}, a_{2}$, and $a_{3}$ are unknown and time varying, but they are upper and lower bounded by unknown constants:

$$
\begin{gathered}
a_{1} \leq \mu_{1}=\frac{B}{\min _{t} J_{e q}} \\
0<\mu_{l 2} \leq a_{2} \leq \mu_{2}=\frac{k_{t}}{\min _{t} J_{e q}}, \\
a_{3} \leq \mu_{3}=\frac{T_{\text {fric }}+\max _{t} T_{L}}{\min _{t} J_{e q}},
\end{gathered}
$$

where $\mu_{1}, \mu_{l 2}, \mu_{2}$, and $\mu_{3}$ are unknown positive constants, and $\min _{t} J_{e q}$ means the minimum value taken for $J_{e q} \forall t$. Similarly $\max _{t} T_{L}$ means the maximum value taken for $T_{L} \forall t$. Assumption (Aii) indicates that the parameters $a_{4}, a_{5}$, and $b$ are unknown constants, and assumption (Aiii) implies that $x_{1}$ and $x_{2}$ must be measured. Now, consider

$$
\begin{gathered}
e(t)=y(t)-y_{d}(t)=W_{m}-y_{d}, \\
\ddot{y}_{d}=-a_{m 1} \dot{y}_{d}-a_{m o} y_{d}+a_{m o} W_{m \mathrm{ref}}, \\
\Omega_{e}=\left\{e:|e| \leq C_{b e}\right\}
\end{gathered}
$$

where $e(t)$ is the tracking error; $y_{d}$ is the desired output $W_{m \text { ref }}$ is the reference value which is user defined; $a_{m 1}, a_{m o}$, and $C_{b e}$ are user-defined positive constants; and $\Omega_{e}$ is a residual set. The objective of the control design is to formulate a control law for the plant model (1) subject to assumptions ( $\mathrm{Ai}$ ) to (Aiii), and such that (Cgi) the tracking error $e$ asymptotically converges to the residual set $\Omega_{e}$, (Cgii) the controller does not involve discontinuous signals, (Cgiii) the control law provides bounded values, and (Cgiv) the closed loops signals are bounded.

\section{Control Design}

In this section a controller for the plant defined by (1) and subject to assumptions (Ai) to (Aiii) is developed taking into account the control goals (Cgi) to (Cgiv) defined previously. The procedure is based on the state adaptive backstepping of [4], but important modifications are introduced in order to handle the unknown time-varying plant model parameters. The controller is developed such that the tracking error $e$ converges to a residual set whose size is user defined. Indeed, the control and update laws are formulated such that the time derivative of the Lyapunov-like function is upper bounded by a function with the following characteristics: (TDi) the function is nonpositive, (TDii) the function is zero if the quadratic form that depends on $z_{1}$ and $z_{2}$ is lower than a prespecified constant size, and (TDiii) the function is negative if such quadratic form is larger than a prespecified constant. If the time derivative of the Lyapunov-like function is upper bounded by a function with such properties, then the asymptotic convergence of the tracking error to a residual set of user-defined size is guaranteed.
Discontinuous signals are avoided in the controller design because such signals may imply (see $[33,34]$ ) loss of trajectory unicity, sliding motion of trajectories along the discontinuity surface that may lead to chattering (see [34, pages 282283]), and input chattering, which is an undesired component of large commutation rate in the control input (see [34, page 292]). Large commutation rate may lead to high power consumption and wear of mechanical components (cf. [35, 36]). Adaptive control based on the direct Lyapunov method involving discontinuous signals needs a rigorous analysis which includes ensuring that trajectory unicity is preserved and developing the Filippov's construction for the case in which sliding motion occurs, in order to avoid chattering. Therefore, it is advisable to avoid discontinuous signals in the controller design.

With the aim to compute the controller the following steps are developed: (i) define the first state $z_{1}$ as the difference between the output and the desired output and differentiate it with respect to time, (ii) define a quadratic function $V_{z_{1}}$ that depends on $z_{1}$ and differentiate it with respect to time; (iii) introduce upper bounds for the time-varying model coefficients, and parameterize them in terms of parameter and regression vectors; (iv) express the parameter vector in terms of updating error and updated parameters, and define the state $z_{2}$; (v) differentiate $z_{2}$ with respect to time, define a quadratic function $V_{z_{2}}$ that depends on $z_{2}$, and differentiate it with respect to time; (vi) introduce upper bounds for the time-varying model coefficients, and parameterize them in terms of parameter and regression vectors; (vii) express the parameter vector in terms of updating error and updated parameter, and formulate the control law; and (viii) formulate the Lyapunov-like function and differentiate it with respect to time, and formulate the update laws.

Step 1. The state variable $z_{1}$ is defined as

$$
z_{1}=e=y-y_{d}=x_{1}-y_{d}=W_{m}-y_{d}
$$

Differentiating (9) with respect to time and using (2) the following is obtained:

$$
\dot{z}_{1}=\dot{x}_{1}-\dot{y}_{d}=-a_{1} x_{1}+a_{2} x_{2}-a_{3}-\dot{y}_{d} .
$$

Step 2. A quadratic form given by

$$
V_{z_{1}}=\left(\frac{1}{2}\right) z_{1}^{2}
$$

is defined. Differentiating (11) with respect to time, using (10), and adding and subtracting $c_{1} z_{1}^{2}$ yields

$$
\begin{aligned}
\dot{V}_{z_{1}} & =z_{1} \dot{z}_{1} \\
& =-c_{1} z_{1}^{2}+z_{1} a_{2} x_{2}+z_{1}\left(-a_{1} x_{1}-a_{3}+c_{1} z_{1}-\dot{y}_{d}\right) .
\end{aligned}
$$

The term $-c_{1} z_{1}^{2}$ has been added to obtain asymptotic convergence of the tracking error later. The unknown time-varying behavior of $a_{1}, a_{2}$, and $a_{3}$ is a significant obstacle for the controller design; for this reason the bounds defined in (5), (6), and (7) will be introduced in Step 3 using the Young's inequality and parameterizations. 
Step 3. Because $a_{1}$ and $a_{3}$ are time varying and unknown, they should be expressed in terms of their upper bounds, and these bounds should be expressed in terms of updated parameters and updating errors. Properties defined in (5) and (7) yield

$$
\begin{aligned}
& z_{1}\left(-a_{1} x_{1}-a_{3}+c_{1} z_{1}-\dot{y}_{d}\right) \\
& \quad \leq \mu_{1}\left|x_{1}\right|\left|z_{1}\right|+\mu_{3}\left|z_{1}\right|+\left|z_{1}\right|\left|c_{1} z_{1}-\dot{y}_{d}\right| .
\end{aligned}
$$

Substituting (13) into (12) and arranging terms the following is obtained:

$$
\dot{V}_{z_{1}} \leq-c_{1} z_{1}^{2}+z_{1}\left(a_{2} x_{2}+\operatorname{sgn}\left(z_{1}\right)\left(\mu_{1}\left|x_{1}\right|+\mu_{3}+\left|c_{1} z_{1}-\dot{y}_{d}\right|\right)\right) .
$$

Equation (14) implies that the possible definition of $z_{2}$ would include the discontinuous term $\operatorname{sgn}\left(z_{1}\right)$. To remedy that the Young's inequality can be applied to (13) such that the $\left|z_{1}\right|$ term leads to $z_{1}^{2}$. Applying the Young's inequality (cf. [37, page 123]) and arranging yield

$$
\begin{aligned}
z_{1}\left(-a_{1} x_{1}-\right. & \left.a_{3}+c_{1} z_{1}-\dot{y}_{d}\right) \\
\leq & \left|a_{1}\right|\left|z_{1}\right|\left|x_{1}\right|+\left|a_{3}\right|\left|z_{1}\right|+\left|z_{1}\right|\left|c_{1} z_{1}-\dot{y}_{d}\right| \\
= & c_{a} \frac{\left|a_{1}\right|\left|z_{1}\right|\left|x_{1}\right|}{c_{a}}+c_{a} \frac{\left|a_{3}\right|\left|z_{1}\right|}{c_{a}}+c_{a} \frac{\left|z_{1}\right|\left|c_{1} z_{1}-\dot{y}_{d}\right|}{c_{a}} \\
\leq & c_{a} \frac{\left|\mu_{1}\right|\left|z_{1}\right|\left|x_{1}\right|}{c_{a}}+c_{a} \frac{\left|\mu_{3}\right|\left|z_{1}\right|}{c_{a}}+c_{a} \frac{\left|z_{1}\right|\left|c_{1} z_{1}-\dot{y}_{d}\right|}{c_{a}} \\
\leq & \frac{c_{a}^{2}}{2}+\frac{1}{2 c_{a}^{2}} \mu_{3}^{2} z_{1}^{2}+\frac{c_{a}^{2}}{2}+\frac{1}{2 c_{a}^{2}} \mu_{1}^{2} x_{1}^{2} z_{1}^{2} \\
& +\frac{c_{a}^{2}}{2}+\frac{1}{2 c_{a}^{2}} z_{1}^{2}\left(c_{1} z_{1}-\dot{y}_{d}\right)^{2} \\
= & 3 \frac{c_{a}^{2}}{2}+\frac{1}{2 c_{a}^{2}} \frac{\mu_{3}^{2}}{\mu_{l 2}} \mu_{l 2} z_{1}^{2}+\frac{1}{2 c_{a}^{2}} \frac{\mu_{1}^{2}}{\mu_{l 2}} \mu_{l 2} x_{1}^{2} z_{1}^{2} \\
& +\frac{1}{2 c_{a}^{2}} \frac{1}{\mu_{l 2}} \mu_{l 2} z_{1}^{2}\left(c_{1} z_{1}-\dot{y}_{d}\right)^{2} .
\end{aligned}
$$

The lower bound $\mu_{l 2}$ and the constant $c_{a}$ have been introduced in order to complete the proof of stability and boundedness of the closed loop signals later. For compactness, the terms involving the unknown constants $\mu_{3}$ and $\mu_{1}$ can be arranged in an unknown constant vector $\theta_{1}$. Substituting (15) into (12) and parameterizing, the following is obtained:

$$
\begin{aligned}
\dot{V}_{z_{1}} \leq & -c_{1} z_{1}^{2}+\frac{3 c_{a}^{2}}{2}+z_{1} a_{2} x_{2} \\
& +\frac{1}{2 c_{a}^{2}} \mu_{l 2} z_{1}^{2}\left(\frac{\mu_{3}^{2}}{\mu_{l 2}}+\frac{\mu_{1}^{2}}{\mu_{l 2}} x_{1}^{2}+\frac{1}{\mu_{l 2}}\left(c_{1} z_{1}-\dot{y}_{d}\right)^{2}\right) \\
= & -c_{1} z_{1}^{2}+\frac{3 c_{a}^{2}}{2}+z_{1} a_{2} x_{2}+\varphi_{1}^{\top} \theta_{1} \frac{1}{2 c_{a}^{2}} \mu_{l 2} z_{1}^{2},
\end{aligned}
$$

where

$$
\begin{gathered}
\varphi_{1}=\left[1, x_{1}^{2},\left(c_{1} z_{1}-\dot{y}_{d}\right)^{2}\right]^{\top}, \\
\theta_{1}=\left[\frac{\mu_{3}^{2}}{\mu_{l 2}}, \frac{\mu_{1}^{2}}{\mu_{l 2}}, \frac{1}{\mu_{l 2}}\right]^{\top},
\end{gathered}
$$

where $\theta_{1}$ is an unknown constant parameter vector and $\varphi_{1}$ is the known regressor vector.

Step 4. Since $\theta_{1}$ is unknown, it should be expressed in terms of updated parameter vector and updating error. The parameter vector $\theta_{1}$ can be rewritten as

$$
\theta_{1}=\widehat{\theta}_{1}-\widetilde{\theta}_{1}
$$

where

$$
\widetilde{\theta}_{1}=\widehat{\theta}_{1}-\theta_{1}=\widehat{\theta}_{1}-\left[\frac{\mu_{3}^{2}}{\mu_{l 2}}, \frac{\mu_{1}^{2}}{\mu_{l 2}}, \frac{1}{\mu_{l 2}}\right]^{\top},
$$

and $\widehat{\theta}_{1}$ is the updated parameter vector provided by the updating law which will be defined later, and $\widetilde{\theta}_{1}$ is the updating error. Substituting (19) into (16) and using (6) yield

$$
\dot{V}_{z_{1}} \leq-c_{1} z_{1}^{2}+\frac{3 c_{a}^{2}}{2}+z_{1} a_{2} x_{2}+\varphi_{1}^{\top} \widehat{\theta}_{1} \frac{1}{2 c_{a}^{2}} \mu_{l 2} z_{1}^{2}-\varphi_{1}^{\top} \tilde{\theta}_{1} \frac{1}{2 c_{a}^{2}} \mu_{l 2} z_{1}^{2}
$$

The updated parameter vector $\widehat{\theta}_{1}$ is nonnegative as will be shown later. Using this fact and (6) it follows that

$$
\dot{V}_{z_{1}} \leq-c_{1} z_{1}^{2}+\frac{3 c_{a}^{2}}{2}+z_{1} a_{2} x_{2}+\varphi_{1}^{\top} \widehat{\theta}_{1} \frac{1}{2 c_{a}^{2}} a_{2} z_{1}^{2}-\varphi_{1}^{\top} \widetilde{\theta}_{1} \frac{1}{2 c_{a}^{2}} \mu_{l 2} z_{1}^{2}
$$

notice that $a_{2}$ is common to the terms involving $x_{2}$ and $\widehat{\theta}_{1}$. Thus, $z_{1} a_{2}$ is as a common factor of those terms and the new state $z_{2}$ is defined by

$$
z_{2}=x_{2}+\varphi_{1}^{\top} \hat{\theta}_{1} \frac{1}{2 c_{a}^{2}} z_{1}
$$

Replacing (23) into (22) yields

$$
\dot{V}_{z_{1}} \leq-c_{1} z_{1}^{2}+\frac{3 c_{a}^{2}}{2}+z_{1} a_{2} z_{2}-\varphi_{1}^{\top} \widetilde{\theta}_{1} \frac{1}{2 c_{a}^{2}} \mu_{l 2} z_{1}^{2}
$$

Remark 2 . The definition of the state $z_{2}$ is significantly different with respect to that of the basic adaptive backstepping scheme of [4]. Indeed, $z_{2}$ involves the vector $\widehat{\theta}_{1}$, and such vector is multiplied by $z_{1}$.

Remark 3. Important modifications have been developed until now, that is, the introduction of the upper bounds of $a_{1}$ and $a_{3}$ (see (13)), the application of the Young's inequality (see (15)), and the parameterization including the constant $\mu_{l 2}$ (see (16)) are some of them. 
Step 5. Differentiating (23) with respect to time, the following is obtained:

$$
\dot{z}_{2}=\dot{x}_{2}+\dot{\varphi}_{1}^{\top} \hat{\theta}_{1} \frac{1}{2 c_{a}^{2}} z_{1}+\varphi_{1}^{\top} \dot{\hat{\theta}}_{1} \frac{1}{2 c_{a}^{2}} z_{1}+\varphi_{1}^{\top} \widehat{\theta}_{1} \frac{1}{2 c_{a}^{2}} \dot{z}_{1},
$$

where $\dot{\varphi}_{1}=\left[0,2 x_{1} \dot{x}_{1}, 2\left(c_{1} z_{1}-\dot{y}_{d}\right)\left(c_{1} \dot{z}_{1}-\ddot{y}_{d}\right)\right]^{\top} . \dot{z}_{2}$ can be rewritten as

$$
\dot{z}_{2}=\dot{x}_{2}+\varphi_{1 b} \dot{x}_{1}+\varphi_{1 c}
$$

where

$$
\begin{aligned}
\varphi_{1 b}=\frac{1}{2 c_{a}^{2}}\left(2\left(x_{1} \widehat{\theta}_{1[2]}+c_{1}\left(c_{1} z_{1}-\dot{y}_{d}\right) \widehat{\theta}_{1[3]}\right) z_{1}+\varphi_{1}^{\top} \widehat{\theta}_{1}\right), \\
\varphi_{1 c}=-2\left(c_{1} z_{1}-\dot{y}_{d}\right)\left(c_{1} \dot{y}_{d}+\ddot{y}_{d}\right) \widehat{\theta}_{1[3]} \frac{1}{2 c_{a}^{2}} z_{1} \\
+\varphi_{1}^{\top} \dot{\hat{\theta}}_{1} \frac{1}{2 c_{a}^{2}} z_{1}-\varphi_{1}^{\top} \widehat{\theta}_{1} \frac{1}{2 c_{a}^{2}} \dot{y}_{d} .
\end{aligned}
$$

Introducing (2) and (3) into (26) yields

$$
\dot{z}_{2}=-a_{4} x_{1}-a_{5} x_{2}+\varphi_{1 b}\left(-a_{1} x_{1}+a_{2} x_{2}-a_{3}\right)+\varphi_{1 c}+b u .
$$

The following quadratic form that depends on $z_{1}$ and $z_{2}$ is chosen:

$$
V_{z}=\left(\frac{1}{2}\right)\left(z_{1}^{2}+z_{2}^{2}\right) .
$$

Differentiating with respect to time and introducing (24) and (28) the following is obtained:

$$
\begin{aligned}
\dot{V}_{z}= & z_{1} \dot{z}_{1}+z_{2} \dot{z}_{2}=\dot{V}_{z_{1}}+z_{2} \dot{z}_{2} \\
\leq & -c_{1} z_{1}^{2}+\frac{3}{2} c_{a}^{2}+z_{1} a_{2} z_{2}-\varphi_{1}^{\top} \widetilde{\theta}_{1} \frac{1}{2 c_{a}^{2}} \mu_{l 2} z_{1}^{2} \\
& +z_{2}\left[-a_{4} x_{1}-a_{5} x_{2}+\varphi_{1 b}\left(-a_{1} x_{1}+a_{2} x_{2}-a_{3}\right)+\varphi_{1 c}+b u\right]
\end{aligned}
$$

The term $-c_{2} z_{2}^{2}$ is added and subtracted in order to obtain asymptotic convergence of the tracking error:

$$
\begin{aligned}
\dot{V}_{z} \leq & -c_{1} z_{1}^{2}-c_{2} z_{2}^{2}+\frac{3}{2} c_{a}^{2} \\
& +z_{2}\left[a_{2} z_{1}-a_{4} x_{1}-a_{5} x_{2}\right. \\
& \left.\quad+\varphi_{1 b}\left(-a_{1} x_{1}+a_{2} x_{2}-a_{3}\right)+\varphi_{1 c}+c_{2} z_{2}+b u\right] \\
& -\varphi_{1}^{\top} \tilde{\theta}_{1} \frac{1}{2 c_{a}^{2}} \mu_{l 2} z_{1}^{2}
\end{aligned}
$$

The control input $u$ is defined as follows:

$$
u=u_{a}+u_{b}
$$

where $u_{a}$ is a user-defined constant. In particular, an adequate choice of $u_{a}$ could prevent saturations of the control input; for this reason the value of $u_{a}$ should be taken from its normal operation range. $u_{b}$ is established by means of the controller design. Substituting (33) into (32) yields

$$
\begin{aligned}
\dot{V}_{z} \leq & -c_{1} z_{1}^{2}-c_{2} z_{2}^{2}+\frac{3}{2} c_{a}^{2} \\
& +z_{2}\left[a_{2} z_{1}-a_{4} x_{1}-a_{5} x_{2}\right. \\
& \left.\quad+\varphi_{1 b}\left(-a_{1} x_{1}+a_{2} x_{2}-a_{3}\right)+b u_{a}+\varphi_{1 c}+c_{2} z_{2}\right] \\
& +b u_{b} z_{2}-\varphi_{1}^{\top} \tilde{\theta}_{1} \frac{1}{2 c_{a}^{2}} \mu_{l 2} z_{1}^{2}
\end{aligned}
$$

Step 6. Since $a_{1}, a_{2}$, and $a_{3}$ are unknown and time varying they should be expressed in terms of their upper bounds. In view of (5), (6), and (7) the term involving the squared brackets can be rewritten as

$$
\begin{aligned}
& z_{2}\left[a_{2} z_{1}-a_{4} x_{1}-a_{5} x_{2}\right. \\
& \left.+\varphi_{1 b}\left(-a_{1} x_{1}+a_{2} x_{2}-a_{3}\right)+b u_{a}+\varphi_{1 c}+c_{2} z_{2}\right] \\
& \leq\left|z_{2}\right|\left(a_{4}\left|x_{1}\right|+a_{5}\left|x_{2}\right|+\mu_{2}\left(\left|z_{1}\right|+\left|x_{2} \varphi_{1 b}\right|\right)\right. \\
& \left.\quad+\mu_{1}\left|x_{1} \varphi_{1 b}\right|+\mu_{3}\left|\varphi_{1 b}\right|+b\left|u_{a}\right|+\left|\varphi_{1 c}+c_{2} z_{2}\right|\right) .
\end{aligned}
$$

Substituting (35) into (34) yields

$$
\begin{aligned}
& \dot{V}_{z} \leq-c_{1} z_{1}^{2}-c_{2} z_{2}^{2}+\frac{3}{2} c_{a}^{2} \\
&+ z_{2}\left[b u_{b}+\operatorname{sgn}\left(z_{2}\right)\right. \\
& \times\left(a_{4}\left|x_{1}\right|+a_{5}\left|x_{2}\right|+\mu_{2}\left(\left|z_{1}\right|+\left|x_{2} \varphi_{1 b}\right|\right)+\mu_{1}\left|x_{1} \varphi_{1 b}\right|\right. \\
&\left.\left.+\mu_{3}\left|\varphi_{1 b}\right|+b\left|u_{a}\right|+\left|\varphi_{1 c}+c_{2} z_{2}\right|\right)\right] \\
&-\varphi_{1}^{\top} \widetilde{\theta}_{1} \frac{1}{2 c_{a}^{2}} \mu_{l 2} z_{1}^{2} .
\end{aligned}
$$

This expression indicates that the possible control rule for $u_{b}$ would involve the discontinuous signal $\operatorname{sgn}\left(z_{2}\right)$. This can be remedied by using the Young's inequality, so that the term $\left|z_{2}\right|$ appearing in the right side of (35) leads to $z_{2}^{2}$. For compactness (35) can be rewritten as

$$
\begin{aligned}
& z_{2}\left[a_{2} z_{1}-a_{4} x_{1}-a_{5} x_{2}+\varphi_{1 b}\left(-a_{1} x_{1}+a_{2} x_{2}-a_{3}\right)\right. \\
& \left.\quad+b u_{a}+\varphi_{1 c}+c_{2} z_{2}\right] \\
& \leq \sqrt{b}\left|z_{2}\right| \bar{\varphi}^{\top} \theta_{2},
\end{aligned}
$$


where

$$
\begin{gathered}
\bar{\varphi}=\left[\left|x_{1}\right|,\left|x_{2}\right|,\left|z_{1}\right|\right. \\
\left.+\left|x_{2} \varphi_{1 b}\right|,\left|x_{1} \varphi_{1 b}\right|,\left|\varphi_{1 b}\right|,\left|u_{a}\right|,\left|\varphi_{1 c}+c_{2} z_{2}\right|\right]^{\top}, \\
\theta_{2}=\frac{1}{\sqrt{b}}\left[a_{4}, a_{5}, \mu_{2}, \mu_{1}, \mu_{3}, b, 1\right]^{\top}
\end{gathered}
$$

$\bar{\varphi}$ is the regression vector whose entries are known, and $\theta_{2}$ is the parameter vector, whose entries are positive, constant, and unknown. The constant $\sqrt{b}$ has been introduced in order to handle the unknown constant parameter $b$ appearing in the term $b u_{b} z_{2}$.

Step 7. Because the parameter vector $\theta_{2}$ is unknown, it should be expressed in terms of updated parameter vector and updating error. The parameter $\theta_{2}$ can be rewritten as

$$
\theta_{2}=\widehat{\theta}_{2}-\widetilde{\theta}_{2}
$$

where

$$
\widetilde{\theta}_{2}=\widehat{\theta}_{2}-\frac{1}{\sqrt{b}}\left[a_{4}, a_{5}, \mu_{2}, \mu_{1}, \mu_{3}, b, 1\right]
$$

where $\widehat{\theta}_{2}$ is the updated parameter vector provided by the update law which will be defined later, and $\widetilde{\theta}_{2}$ is the updating error. Substituting (39) into (37) yields

$$
\begin{gathered}
z_{2}\left[a_{2} z_{1}-a_{4} x_{1}-a_{5} x_{2}+\varphi_{1 b}\left(-a_{1} x_{1}+a_{2} x_{2}-a_{3}\right)\right. \\
\left.\quad+b u_{a}+\varphi_{1 c}+c_{2} z_{2}\right] \\
\leq \sqrt{b}\left|z_{2}\right| \bar{\varphi}^{\top} \widehat{\theta}_{2}-\sqrt{b}\left|z_{2}\right| \bar{\varphi}^{\top} \widetilde{\theta}_{2} .
\end{gathered}
$$

Arranging the term $\sqrt{b}\left|z_{2}\right| \bar{\varphi}^{\top} \widehat{\theta}_{2}$ and applying the Young's inequality (cf. [37, page 123]) yield

$$
\sqrt{b}\left|z_{2}\right| \bar{\varphi}^{\top} \widehat{\theta}_{2}=c_{c} \frac{1}{c_{c}} \sqrt{b}\left|z_{2}\right| \bar{\varphi}^{\top} \widehat{\theta}_{2} \leq \frac{c_{c}^{2}}{2}+\frac{1}{2 c_{c}^{2}} b z_{2}^{2}\left(\bar{\varphi}^{\top} \widehat{\theta}_{2}\right)^{2} .
$$

The constant $c_{c}$ is added to prove the stability. Substituting (42) into (41) yield

$$
\begin{gathered}
z_{2}\left[a_{2} z_{1}-a_{4} x_{1}-a_{5} x_{2}+\varphi_{1 b}\left(-a_{1} x_{1}+a_{2} x_{2}-a_{3}\right)\right. \\
\left.+b u_{a}+\varphi_{1 c}+c_{2} z_{2}\right] \\
\leq \frac{c_{c}^{2}}{2}+\frac{1}{2 c_{c}^{2}} b z_{2}^{2}\left(\bar{\varphi}^{\top} \widehat{\theta}_{2}\right)^{2}-\sqrt{b}\left|z_{2}\right| \bar{\varphi}^{\top} \widetilde{\theta}_{2} .
\end{gathered}
$$

Substituting (43) into (34) and arranging yield

$$
\begin{aligned}
\dot{V}_{z} \leq & -c_{1} z_{1}^{2}-c_{2} z_{2}^{2}+\frac{3}{2} c_{a}^{2}+\frac{c_{c}^{2}}{2} \\
& +b z_{2}\left(u_{b}+\frac{1}{2 c_{c}^{2}} z_{2}\left(\bar{\varphi}^{\top} \widehat{\theta}_{2}\right)^{2}\right) \\
& -\varphi_{1}^{\top} \widetilde{\theta}_{1} \frac{1}{2 c_{a}^{2}} \mu_{l 2} z_{1}^{2}-\sqrt{b}\left|z_{2}\right| \bar{\varphi}^{\top} \widetilde{\theta}_{2} .
\end{aligned}
$$

The following expression can be used for $u_{b}$ :

$$
u_{b}=-\frac{1}{2 c_{c}^{2}} z_{2}\left(\bar{\varphi}^{\top} \hat{\theta}_{2}\right)^{2}
$$

In view of (33), the control law for $u$ is

$$
u=u_{a}-\frac{1}{2 c_{c}^{2}} z_{2}\left(\bar{\varphi}^{\top} \hat{\theta}_{2}\right)^{2}
$$

Substituting (46) into (44) the following is obtained:

$$
\begin{aligned}
\dot{V}_{z} \leq & -2 \min \left\{c_{1}, c_{2}\right\} V_{z}+\frac{3}{2} c_{a}^{2}+\frac{c_{c}^{2}}{2} \\
& -\varphi_{1}^{\top} \tilde{\theta}_{1} \frac{1}{2 c_{a}^{2}} \mu_{l 2} z_{1}^{2}-\sqrt{b}\left|z_{2}\right| \bar{\varphi}^{\top} \tilde{\theta}_{2}
\end{aligned}
$$

To handle the effect of the constant $(3 / 2) c_{a}^{2}+(1 / 2) c_{c}^{2}$, the following quadratic-like function is defined:

$$
\begin{gathered}
\bar{V}_{z}= \begin{cases}\left(\frac{1}{2}\right)\left(\sqrt{V_{z}}-\sqrt{C_{b v z}}\right)^{2} & \text { if } V_{z} \geq C_{b v z} \\
0 & \text { otherwise }\end{cases} \\
C_{b v z}=\left(\frac{1}{2}\right) C_{b e}^{2} .
\end{gathered}
$$

The term $V_{z}$ is defined in (29). Function defined by (48) and (49) has the following properties:

$$
\begin{gathered}
\bar{V}_{z} \geq 0 \\
V_{z} \leq 3 C_{b v z}+3 \bar{V}_{z}, \\
\bar{V}_{z}, \frac{\partial \bar{V}_{z}}{\partial V_{z}} \text { are locally Lipschitz continuous. }
\end{gathered}
$$

Differentiating (48) with respect to time the following is obtained:

$$
\frac{d \bar{V}_{z}}{d t}=\frac{\partial \bar{V}_{z}}{\partial V_{z}} \dot{V}_{z}
$$

where

$$
\frac{\partial \bar{V}_{z}}{\partial V_{z}}= \begin{cases}\frac{1}{2} \frac{\sqrt{V_{z}}-\sqrt{C_{b v z}}}{\sqrt{V_{z}}}, & \text { if } V_{z} \geq C_{b v z} \\ 0, & \text { otherwise }\end{cases}
$$

From (52) it follows that $\partial \bar{V}_{z} / \partial V_{z}$ is nonnegative, so that it can be introduced in both sides of (47) without changing the sense of the inequality:

$$
\begin{aligned}
\frac{\partial \bar{V}_{z}}{\partial V_{z}} \dot{V}_{z} \leq & -2 \min \left\{c_{1}, c_{2}\right\} V_{z} \frac{\partial \bar{V}_{z}}{\partial V_{z}}+\left(\frac{3}{2} c_{a}^{2}+\frac{c_{c}^{2}}{2}\right) \frac{\partial \bar{V}_{z}}{\partial V_{z}} \\
& -\varphi_{1}^{\top} \widetilde{\theta}_{1} \frac{1}{2 c_{a}^{2}} \mu_{l 2} z_{1}^{2} \frac{\partial \bar{V}_{z}}{\partial V_{z}}-\sqrt{b}\left|z_{2}\right| \bar{\varphi}^{\top} \widetilde{\theta}_{2} \frac{\partial \bar{V}_{z}}{\partial V_{z}}
\end{aligned}
$$


Combining (51) and (53) yields

$$
\begin{aligned}
\frac{d \bar{V}_{z}}{d t} \leq & -2 \min \left\{c_{1}, c_{2}\right\} V_{z} \frac{\partial \bar{V}_{z}}{\partial V_{z}}+\left(\frac{3}{2} c_{a}^{2}+\frac{c_{c}^{2}}{2}\right) \frac{\partial \bar{V}_{z}}{\partial V_{z}} \\
& -\varphi_{1}^{\top} \widetilde{\theta}_{1} \frac{1}{2 c_{a}^{2}} \mu_{l 2} z_{1}^{2} \frac{\partial \bar{V}_{z}}{\partial V_{z}}-\sqrt{b}\left|z_{2}\right| \bar{\varphi}^{\top} \widetilde{\theta}_{2} \frac{\partial \bar{V}_{z}}{\partial V_{z}}
\end{aligned}
$$

Step 8. The following Lyapunov-like function is defined:

$$
\begin{gathered}
V=\bar{V}_{z}+V_{\theta} \\
V_{\theta}=\left(\frac{1}{2}\right) \mu_{l 2} \widetilde{\theta}_{1}^{\top} \Gamma_{1}^{-1} \widetilde{\theta}_{1}+\left(\frac{1}{2}\right) \sqrt{b} \widetilde{\theta}_{2}^{\top} \Gamma_{2}^{-1} \widetilde{\theta}_{2}
\end{gathered}
$$

where $\widetilde{\theta}_{1}$ and $\widetilde{\theta}_{2}$ are defined in (20) and (40), respectively. To compute $\dot{V}$, the time derivative of $V_{\theta}$ is computed as

$$
\begin{aligned}
\dot{V}_{\theta} & =\frac{\mu_{l 2}}{2}\left\{\dot{\tilde{\theta}}_{1}^{\top} \Gamma_{1}^{-1} \widetilde{\theta}_{1}+\widetilde{\theta}_{1} \Gamma_{1}^{-1} \dot{\tilde{\theta}}_{1}\right\}+\frac{\sqrt{b}}{2}\left\{\dot{\tilde{\theta}}_{2}^{\top} \Gamma_{2}^{-1} \widetilde{\theta}_{2}+\widetilde{\theta}_{2} \Gamma_{2}^{-1} \dot{\widetilde{\theta}}_{2}\right\} \\
& =\mu_{l 2} \widetilde{\theta}_{1}^{\top} \Gamma_{1}^{-1} \dot{\widehat{\theta}}_{1}+\sqrt{b} \widetilde{\theta}_{2}^{\top} \Gamma_{2}^{-1} \dot{\hat{\theta}}_{2} .
\end{aligned}
$$

Now, differentiating (55) with respect to time and using (54) and (57) yield

$$
\begin{aligned}
\dot{V}= & \dot{\bar{V}}_{z}+\dot{V}_{\theta} \leq-2 \min \left\{c_{1}, c_{2}\right\} V_{z} \frac{\partial \bar{V}_{z}}{\partial V_{z}}+\left(\frac{3}{2} c_{a}^{2}+\frac{c_{c}^{2}}{2}\right) \frac{\partial \bar{V}_{z}}{\partial V_{z}} \\
& +\mu_{l 2} \widetilde{\theta}_{1}^{\top}\left(-\varphi_{1} \frac{1}{2 c_{a}^{2}} z_{1}^{2} \frac{\partial \bar{V}_{z}}{\partial V_{z}}+\Gamma_{1}^{-1} \dot{\hat{\theta}}_{1}\right) \\
& +\sqrt{b} \widetilde{\theta}_{2}^{\top}\left(-\left|z_{2}\right| \bar{\varphi} \frac{\partial \bar{V}_{z}}{\partial V_{z}}+\Gamma_{2}^{-1} \dot{\hat{\theta}}_{2}\right) .
\end{aligned}
$$

To tackle the effect of the terms involving the updating errors $\widetilde{\theta}_{1}$ and $\widetilde{\theta}_{2}$ the following update laws are formulated:

$$
\begin{gathered}
\dot{\hat{\theta}}_{1}=\Gamma_{1} \varphi_{1} \frac{1}{2 c_{a}^{2}} z_{1}^{2} \frac{\partial \bar{V}_{z}}{\partial V_{z}} \quad \text { with } \widehat{\theta}_{1}\left(t_{0}\right) \geq 0, \\
\dot{\hat{\theta}}_{2}=\Gamma_{2}\left|z_{2}\right| \bar{\varphi} \frac{\partial \bar{V}_{z}}{\partial V_{z}} \quad \text { with } \widehat{\theta}_{2}\left(t_{0}\right) \geq 0,
\end{gathered}
$$

where $\Gamma_{1}$ and $\Gamma_{2}$ are diagonal matrices whose elements are user-defined positive constants. From (17) and (52) it follows that $\varphi_{1}=\left|\varphi_{1}\right| \geq 0$ and $\partial \bar{V}_{z} / \partial V_{z} \geq 0$ such that $\widehat{\theta}_{1}(t)=\left|\widehat{\theta}_{1}\right| \geq 0$. Substituting (59) into (58) yields

$$
\begin{aligned}
\dot{V} & \leq-\frac{\partial \bar{V}_{z}}{\partial V_{z}}\left(2 \min \left\{c_{1}, c_{2}\right\} V_{z}-\frac{3}{2} c_{a}^{2}-\frac{c_{c}^{2}}{2}\right) \\
& =-\min \left\{c_{1}, c_{2}\right\} \frac{\partial \bar{V}_{z}}{\partial V_{z}}\left(2 V_{z}-\frac{1}{2} \frac{3 c_{a}^{2}+c_{c}^{2}}{\min \left\{c_{1}, c_{2}\right\}}\right)
\end{aligned}
$$

Although the control law (46) and the update laws (59) have been formulated, the values of the constants $c_{a}$ and $c_{c}$ have not been defined. The constants $c_{a}$ and $c_{c}$ are positive constants defined by the user and they must satisfy

$$
3 c_{a}^{2}+c_{c}^{2} \leq 2 \min \left\{c_{1}, c_{2}\right\} C_{b v z}
$$

A simple choice that satisfies the above requirement is

$$
c_{a}=c_{c}=\left(\frac{1}{2}\right) C_{b e} \sqrt{\min \left\{c_{1}, c_{2}\right\}}
$$

From (52) the following is obtained:

$$
\begin{aligned}
& \frac{\partial \bar{V}_{z}}{\partial V_{z}}=0 \text { for } V_{z} \leq C_{b v z} \\
& \frac{\partial \bar{V}_{z}}{\partial V_{z}}>0 \text { for } V_{z}>C_{b v z} .
\end{aligned}
$$

From (63), (61), and (60) it follows that

$$
\begin{gathered}
\dot{V} \leq 0=-c_{1} V_{z} \frac{\partial \bar{V}_{z}}{\partial V_{z}}, \quad \text { if } V_{z} \leq C_{b v z}, \\
\dot{V} \leq-\min \left\{c_{1}, c_{2}\right\} \frac{\partial \bar{V}_{z}}{\partial V_{z}} V_{z} \leq 0, \quad \text { if } V_{z} \geq C_{b v z} .
\end{gathered}
$$

Finally, the combination of the above expressions yields

$$
\dot{V} \leq-\min \left\{c_{1}, c_{2}\right\} \frac{\partial \bar{V}_{z}}{\partial V_{z}} V_{z}
$$

The developed controller involves the control law (46) and the update laws (59). The signals and parameters necessary to implement it are $z_{1}, z_{2}, \varphi_{1}, \bar{\varphi}, \varphi_{1 b}, \varphi_{1 c}, \partial \bar{V}_{z} / \partial V_{z}, V_{z}$, and $\bar{V}_{z}$ which are given by (9), (23), (17), (38), (27), (52), (29), and (48), respectively. In addition, $c_{1}, c_{2}$, the diagonal elements of $\Gamma_{1}$ and $\Gamma_{2}, c_{a}, c_{c}$, and $u_{a}$ are user-defined positive constants. In particular, $c_{a}$ and $c_{c}$ must satisfy $(61), C_{b v z}=(1 / 2) C_{b e}^{2}$ where $C_{b e}$ is a user-defined positive constant.

Remark 4. Expression (65) indicates that $\dot{V}$ fulfills conditions (TDi), (TDii), and (TDiii) mentioned at the beginning of Section 3.

Remark 5. The developed controller has the following benefits. (i) it does not use upper or lower bounds of coefficients of model (2) and (3). Indeed, the controller does not use any of the constants $b_{m x}, \mu_{l 1}, \mu_{1}, \mu_{2}, \mu_{3}$, and $\mu_{4}$. This implies less modeling effort. (ii) It does not involve discontinuous signals. This implies that the vector field of the closed loop system is locally Lipschitz continuous, so that trajectory unicity is preserved and sliding motion is absent according to [33]. The locally Lipschitz nature of $\partial \bar{V}_{z} / \partial V_{z}$ is important to avoid discontinuous signals in the update law. 
Remark 6. The main elements to handle the unknown varying nature of coefficients $a_{1}, a_{2}$, and $a_{3}$ are as follows: (i) introduce the constant $\mu_{l 2}$ in the parametrization in (16), (17), and (18); (ii) introduce the relationship between $\mu_{l 2}$ and $a_{2}$ provided by (6) in (24); (iii) express $a_{1}, a_{2}$, and $a_{3}$ in terms of their upper bounds in (35); (iv) introduce the constant $\sqrt{b}$ in the unknown parameter vector $\theta_{2}$ (see (37) and (38)); (v) apply the Young's inequality to the term $\sqrt{b}\left|z_{2}\right| \bar{\varphi}^{\top} \hat{\theta}_{2}$ (see (42)); and (vi) formulate the function $\bar{V}_{z}$ which is a truncated version of the quadratic form $V_{z}$ (see (48)). The vanishing of $\partial \bar{V}_{z} / \partial V_{z}$ allows preserving the decreasing nature of the Lyapunov-like function, as can be noticed from (65). The continuous nature of the derivative of $\bar{V}_{z}$ with respect to $V_{z}$ allows to avoid discontinuous signals in the update law.

Remark 7. The effect of a low value of $C_{b e}$ is analyzed at the following. From (59), (52), (49), and (61) it follows that a low value of $C_{b e}$ implies the following facts: (i) the term $\partial \bar{V}_{z} / \partial V_{z}$ is nonzero for longer time lapses, and consequently the update law (59) is active for longer time lapses; the updated parameter $\widehat{\theta}_{2}$ increases during longer time lapses, which leads to reach larger values; (ii) the constant $C_{b v z}$ is smaller, and consequently the values of $\sqrt{V_{z}}-\sqrt{C_{b v z}}, \partial \bar{V}_{z} / \partial V_{z}, \dot{\hat{\theta}}_{2}$, and $\widehat{\theta}_{2}$ increase; and (iii) the chosen values of $c_{a}$ and $c_{c}$ have to be lower in order to accomplish condition (61). Therefore, the term $c_{c}^{-2}\left(\bar{\varphi}^{\top} \hat{\theta}_{2}\right)^{2}$ becomes larger and from (46) it follows that $u-u_{a}$ takes on larger values, which is interpreted as a bigger control effort.

The developed controller achieves some of the benefits mentioned in introduction. Sections 4.1 and 4.2 complete the proof of the other proposed benefits.

\section{Boundedness Analysis}

In this section it is proven that the closed loop signals are bounded if the developed controller is used; also the convergence of the tracking error to a residual set is proven.

\subsection{Boundedness of the Closed Loop Signals}

Theorem 8 (boundedness of the closed loop signals). Consider the plant model given by (1) which is subject to assumptions (Ai) to (Aiii). The signals $z_{1}$ and $z_{2}$ are defined in (9) and (23); $\varphi_{1}, \varphi_{1 b}, \varphi_{1 c}$, and $\bar{\varphi}$ are defined in (17), (27), and (38), respectively; the signals $V_{z}, \bar{V}_{z}$, and $\partial \bar{V}_{z} / \partial V_{z}$ are defined in (29), (48), and (52), respectively; the constant $C_{b v z}$ is defined by (49) and the constants $c_{a}$ and $c_{c}$ satisfy (61). If the controller defined in (46) and (59) is applied, then the signals $z_{1}, z_{2}, \widehat{\theta}_{1}$, $\widehat{\theta}_{2}$, and $u$ remain bounded.

Proof. From (65) it follows that

$$
\dot{V} \leq 0, \quad V(x(t)) \leq V\left(x\left(t_{o}\right)\right),
$$

where

$$
\begin{gathered}
V\left(x\left(t_{o}\right)\right)=\bar{V}_{z o}+V_{\theta o}, \\
\bar{V}_{z o}= \begin{cases}\left(\frac{1}{2}\right)\left(\sqrt{V_{z o}}-\sqrt{C_{b v z}}\right) & \text { if } V_{z o} \geq C_{b v z}, \\
0 & \text { otherwise, }\end{cases} \\
V_{z o}=\left(\frac{1}{2}\right)\left(z_{1}\left(t_{o}\right)^{2}+z_{2}\left(t_{o}\right)^{2}\right), \\
V_{\theta o}=\left(\frac{1}{2}\right) \mu_{l 1}\left(\widehat{\theta}_{1}\left(t_{o}\right)-\theta_{1}\right)^{\top} \Gamma_{1}^{-1}\left(\widehat{\theta}_{1}\left(t_{o}\right)-\theta_{1}\right) \\
+\left(\frac{1}{2}\right) \sqrt{b}\left(\widehat{\theta}_{2}\left(t_{o}\right)-\theta_{2}\right)^{\top} \Gamma_{2}^{-1}\left(\widehat{\theta}_{2}\left(t_{o}\right)-\theta_{2}\right) .
\end{gathered}
$$

From (66) and (55) it follows that

$$
\bar{V}_{z}+V_{\theta} \leq V\left(x\left(t_{o}\right)\right), \quad \bar{V}_{z} \leq V\left(x\left(t_{o}\right)\right), V_{\theta} \leq V\left(x\left(t_{o}\right)\right) .
$$

Using (56) the following is obtained: $\widetilde{\theta}_{1} \in L_{\infty}$ and $\widetilde{\theta}_{2} \in L_{\infty}$; consequently $\widehat{\theta}_{1} \in L_{\infty}$ and $\widehat{\theta}_{2} \in L_{\infty}$. The upper bound for the tracking error is defined as follows. Solving (48) for $V_{z}$ yields

$$
\begin{gathered}
V_{z}=\left(\sqrt{2 \bar{V}_{z}}+\sqrt{C_{b v z}}\right)^{2}, \quad \text { if } \bar{V}_{z}>0 \\
V_{z} \leq C_{b v z}, \quad \text { otherwise. }
\end{gathered}
$$

Using (71) yields

$$
\begin{gathered}
V_{z} \leq\left(\sqrt{2 V\left(x\left(t_{o}\right)\right)}+\sqrt{C_{b v z}}\right)^{2}, \quad \text { if } \bar{V}_{z}>0, \\
V_{z} \leq C_{b v z} \leq\left(\sqrt{2 V\left(x\left(t_{o}\right)\right)}+\sqrt{C_{b v z}}\right)^{2}, \quad \text { if } \bar{V}_{z}=0 .
\end{gathered}
$$

Both expressions of (73) can be combined to obtain

$$
V_{z} \leq\left(\sqrt{C_{b v z}}+\sqrt{2 V\left(x\left(t_{o}\right)\right)}\right)^{2} .
$$

Introducing (29) yields

$$
\sqrt{z_{1}^{2}+z_{2}^{2}} \leq \sqrt{2}\left(\sqrt{C_{b v z}}+\sqrt{2 V\left(x\left(t_{o}\right)\right)}\right)
$$

where $V\left(x\left(t_{o}\right)\right)$ is defined in (67). Therefore $z_{1} \in L_{\infty}$ and $z_{2} \in L_{\infty}$. Because $e=z_{1}$, then

$$
|e| \leq \sqrt{z_{1}^{2}+z_{2}^{2}} \leq \sqrt{2}\left(\sqrt{C_{b v z}}+\sqrt{2 V\left(x\left(t_{o}\right)\right)}\right),
$$

which is an upper bound for the transient behavior of the tracking error $e$.

In the following it is proven that $u$ is bounded. From (9), (23), $z_{1} \in L_{\infty}, z_{2} \in L_{\infty}, \widehat{\theta}_{1} \in L_{\infty}$, and $\widehat{\theta}_{2} \in L_{\infty}$ it follows that $x_{1} \in L_{\infty}$ and $x_{2} \in L_{\infty}$. Therefore from (17), (27), and (38) it follows that $\varphi_{1} \in L_{\infty}, \varphi_{1 b} \in L_{\infty}, \varphi_{1 c} \in L_{\infty}$, and $\bar{\varphi} \in L_{\infty}$. Finally from (46) it follows that $u \in L_{\infty}$.

Remark 9. Notice that the upper bound of (76) does not involve integral terms, which is an important advantage with respect to controllers that involve the Nussbaum gain method (see $[20,21])$. 


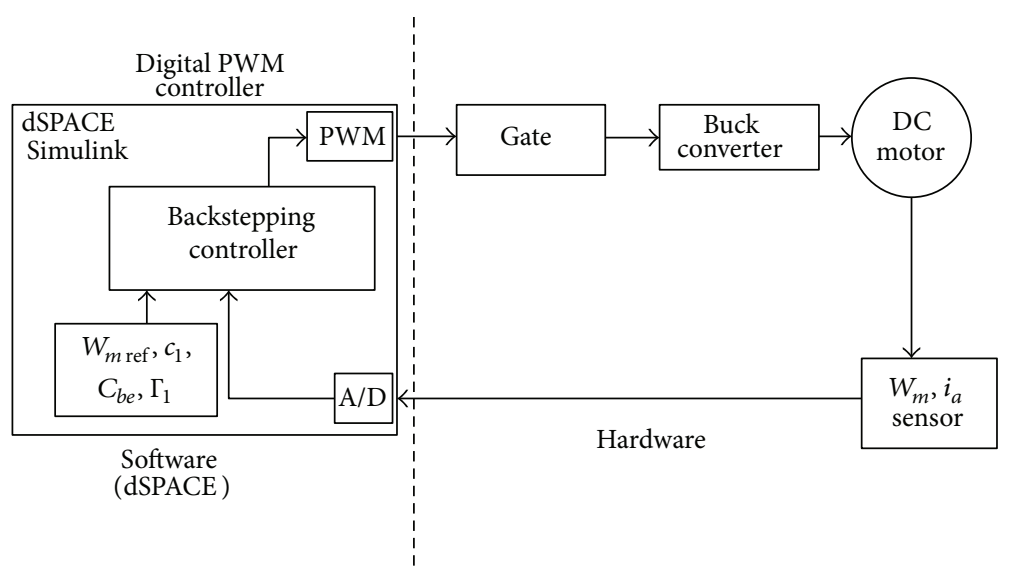

FIGURE 1: Block diagram of the proposed system.

4.2. Convergence Analysis. Now it is proven that the developed controller induces asymptotic convergence of the tracking error $e$ to the residual set $\Omega_{e}$, where $\Omega_{e}=\left\{e:|e| \leq C_{b e}\right\}$, with $C_{b e}$ defined by the user.

Theorem 10 (convergence of the tracking error). Consider the plant model given by (1) which is subject to assumptions (Ai) to (Aiii); the signals $z_{1}, z_{2}, \varphi_{1}, \varphi_{1 b}, \varphi_{1 c}, \bar{\varphi}, V_{z}, \bar{V}_{z}$, and $\partial \bar{V}_{z} / \partial V_{z}$ are defined by (9), (23), (17), (27), (38), (29), (48), and (52), respectively; the constant $C_{b v z}$ is defined in (49) and the constants $c_{a}, c_{c}$ satisfy (61). If the controller given by (46) and (59) is applied, then the tracking error e asymptotically converges to a residual set $\Omega_{e}$, where $\Omega_{e}=\left\{e:|e| \leq C_{b e}\right\}$.

Proof. In view of (52), inequality (65) can be rewritten as

$$
\begin{gathered}
\dot{V} \leq-c_{1} f_{d} \leq 0, \\
f_{d}= \begin{cases}\left(\frac{1}{2}\right)\left(\sqrt{V_{z}}-\sqrt{C_{b v z}}\right) \sqrt{V_{z}}, & \text { if } V_{z} \geq C_{b v z}, \\
0, & \text { otherwise. }\end{cases}
\end{gathered}
$$

It can be noticed that the term $\partial f_{d} / \partial V_{z}$ is not continuous, because it involves an abrupt change at $V=C_{b v z}$; for this reason the Barbalat's lemma cannot be applied on $f_{d}$. To remedy that, (77) can be expressed in terms of a function with continuous derivative as follows:

$$
\dot{V} \leq-c_{1} f_{d} \leq-c_{1} f_{g} \leq 0 \quad \forall t \geq t_{o},
$$

where

$$
f_{g}= \begin{cases}\left(\frac{1}{2}\right)\left(\sqrt{V_{z}}-\sqrt{C_{b v z}}\right)^{2}, & \text { if } V_{z} \geq C_{b v z} \\ 0, & \text { otherwise. }\end{cases}
$$

Arranging and integrating (79) the following is obtained:

$$
\begin{aligned}
& c_{1} \int_{t_{o}}^{t} f_{g} d \tau \leq V\left(x\left(t_{o}\right)\right)-V(x(t)), \\
& V(x(t))+c_{1} \int_{t_{o}}^{t} f_{g} d \tau \leq V\left(x\left(t_{o}\right)\right) .
\end{aligned}
$$

Therefore $f_{g} \in L_{1}$. In order to apply the Barbalat's lemma it is necessary to prove that $f_{g} \in L_{\infty}$ and $\dot{f}_{g} \in L_{\infty}$. Since $V_{z} \in L_{\infty}$ it follows from (80) that $f_{g} \in L_{\infty}$. Differentiating (80) with respect to time yields

$$
\begin{gathered}
\dot{f}_{g}=\frac{\partial f_{g}}{\partial V_{z}} \dot{V}_{z}, \\
\frac{\partial f_{g}}{\partial V_{z}}= \begin{cases}\frac{\sqrt{V_{z}}-\sqrt{C_{b v z}}}{2 \sqrt{V_{z}}}, & \text { if } V_{z} \geq C_{b v z}, \\
0, & \text { otherwise. }\end{cases}
\end{gathered}
$$

Notice that $\partial f_{g} / \partial V_{z}$ is continuous with respect to $V_{z}$. Since $V_{z} \in L_{\infty}$ then $\partial f_{g} / \partial V_{z} \in L_{\infty}$. Because $z_{1} \in L_{\infty}, z_{2} \in$ $L_{\infty}, x_{1} \in L_{\infty}, x_{2} \in L_{\infty}$, and $u \in L_{\infty}$ it follows from (10) and (25) that $\dot{z}_{1} \in L_{\infty}$ and $\dot{z}_{2} \in L_{\infty}$. Thus, from (30) it follows that $\dot{V}_{z} \in L_{\infty}$. Because $\partial f_{g} / \partial V_{z} \in L_{\infty}$ and $\dot{V}_{z} \in L_{\infty}$ then it follows from (82) that $\dot{f}_{g} \in L_{\infty}$. Because $f_{g} \in L_{\infty}$ and $\dot{f}_{g} \in L_{\infty}$ the Barbalat's lemma (cf. [38, page 76]) indicates that $f_{g}$ asymptotically converges to zero. From (80) it follows that $V_{z}$ converges to $\Omega_{v z}$, where $\Omega_{v z}=\left\{V_{z}: V_{z} \leq C_{b v z}\right\}$. Furthermore, from (29) it follows that $z_{1}$ asymptotically converges to $\Omega_{z_{1}}$, where $\Omega_{z_{1}}=\left\{z_{1}\right.$ : $\left.\left|z_{1}\right| \leq \sqrt{2 C_{b v z}}\right\}$. Since $C_{b v z}=(1 / 2) C_{b e}^{2}$ and $z_{1}=e$, then $e$ asymptotically converges to $\Omega_{e}$, where $\Omega_{e}=\{e:|e| \leq$ $\left.C_{b e}\right\}$.

Remark 11. The tracking error $e$ converges to a residual set whose size is user defined and not altered by the varying parameters.

\section{Numerical and Experimental Results}

In this section numerical and experimental results are shown. Figure 1 shows the block diagram of the system under study. This system is divided into two major groups: the first one is composed by all hardware parts, including physical and electronic components; the second one is related to software and is implemented in a dSPACE platform, where the acquisition signals and the control technique are performed. 
The hardware is composed of a permanent magnet DC motor (PMDC) with rated power 250 Watts, rated Volts 42 VDC, rated current 6 Amps, and 4000 RPM of maximum speed. For the acquisition of motor speed $W_{m}$, a 1000 pulses per turn encoder was used. A series resistance was used to measure the armature current $\left(i_{a}\right)$. The digital part and the backstepping control technique are implemented in the control and development card dSPACE DS1104. This card is programmed from Matlab/Simulink platform and it has a graphical display interface called ControlDesk. The controller is implemented in Simulink and is downloaded to the DSP. The sampling rate for all variables $\left(W_{m}\right.$ and $\left.i_{a}\right)$ is set to $4 \mathrm{kHz}$. The state variable $i_{a}$ is 12 -bit resolution; the controlled variable $W_{m}$ is sensed by an encoder which has 28-bit resolution and the duty cycle $(d)$ is 10-bit resolution. At each sampling time ( $250 \mu \mathrm{s})$ the controller uses the measured $W_{m}$ and $i_{a}$ to calculate the duty cycle $d$, as follows: (i) the control input $u=v_{c}$ is determined according to the control and update laws based on the proposed procedure (see Section 4), (ii) the duty cycle $d$ is computed from $d=u / E$, and (iii) the duty cycle $d$ is transformed into a PWMC pulse signal. To obtain simulation results the parameters of DC motor $\left(B, J_{e q}\right.$, $k_{t}, k_{e}, T_{\text {fric }}, R_{a}$, and $\left.L_{a}\right)$ and backstepping controller $\left(C_{b e}, c_{1}\right.$, $c_{2}, \Gamma_{1}, \Gamma_{2}, u_{a}$, and $\left.W_{m \text { ref }}\right)$ are entered to the control block by the user, as constant parameters. The load torque $T_{L}$ is time varying and unknown.

Figure 2 shows a sketch of the Simulink benchmark. The controller uses the measurements of $W_{m}$ and $i_{a}$ to compute $u=v_{c}$, the duty cycle applied to buck converter is given by $d=u / E$. The zero-order hold, quantizer, and delay are included in order to model the signal acquisition and the analog to digital signal conversion. The motor and buck converter parameters used in simulation were experimentally measured and are presented in Table 1. Recall that the values of the motor parameters $\left(R_{a}, L_{a}, B, J_{e q}, k_{t}, k_{e}, T_{\text {fric }}\right.$, and $\left.T_{L}\right)$ and the converter parameters $\left(E, V_{f d}, r_{s}, r_{L}, C\right.$, and $\left.L\right)$ are not used by the control or update laws, neither in simulation nor experimentation.

Figure 3 shows the desired output $y_{d}$ and the measured and simulated output $W_{m}$ when $W_{m \text { ref }}=200 \mathrm{rad} / \mathrm{s}$. In Figures 4(a) and 4(b), the numerical and experimental tracking errors $e=W_{m}-y_{d}$ are shown. It can be seen that in simulation the error converges to a residual set $\Omega_{e}=5 \mathrm{rad} / \mathrm{s}$ (whose size is given by $C_{b e}=5$ ) and in experiment there is a bit error, probably due to quantization effects, delays, or unmodeled dynamics. Nevertheless, experimental and numerical results agree.

Figure 5 shows the numerical and experimental controller performance when $W_{m \text { ref }}$ changes from $200 \mathrm{rad} / \mathrm{s}$ to $300 \mathrm{rad} / \mathrm{s}$ at $t=1 \mathrm{~s}$. As in previous example, $|e|<5 \mathrm{rad} / \mathrm{s}$ in steady state for simulation case and in experimental case the results are very close to this bound.

Figure 6 shows the results when the load torque $T_{L}+T_{\text {fric }}$ changes from $0.1639 \mathrm{~N} \cdot \mathrm{m}$ to $0.101 \mathrm{~N} \cdot \mathrm{m}$ at $t=1 \mathrm{~s}$. Notice that the controller achieves successfully tracking error, and the steady state bound is very close to the given value $C_{b e}=5$. An estimator for the torque was added only with the aim to show simulation and real values of load torque, but this estimator does not work in the controller.
TABLE 1: Simulation and experimentation parameters.

\begin{tabular}{|c|c|}
\hline Parameter & Value \\
\hline$E:$ input voltage & $40 \mathrm{~V}$ \\
\hline $\begin{array}{l}r_{s}: \text { internal resistance of the source and } \\
\text { MOSFET }\end{array}$ & $0.84 \Omega$ \\
\hline$V_{f d}:$ diode forward voltage & $1.1 \mathrm{~V}$ \\
\hline$L:$ inductance & $2.473 \mathrm{mH}$ \\
\hline$r_{L}:$ internal resistance of the inductor & $1.695 \Omega$ \\
\hline C: capacitance & $46.27 \mu \mathrm{F}$ \\
\hline$R_{a}:$ armature resistance & $2.7289 \Omega$ \\
\hline$L_{a}:$ armature inductance & $1.17 \mathrm{mH}$ \\
\hline$B$ : viscous friction coefficient & $0.000138 \mathrm{~N} \cdot \mathrm{m} / \mathrm{rad} / \mathrm{s}$ \\
\hline$J_{e q}:$ moment of inertia & $0.000115 \mathrm{~kg} \cdot \mathrm{m}^{2}$ \\
\hline$k_{t}:$ motor torque constant & $0.0663 \mathrm{~N} \cdot \mathrm{m} / \mathrm{A}$ \\
\hline$k_{e}:$ voltage constant & $0.0663 \mathrm{~V} / \mathrm{rad} / \mathrm{s}$ \\
\hline$T_{\text {fric }}:$ friction torque & $0.0284 \mathrm{~N} \cdot \mathrm{m}$ \\
\hline$T_{L}:$ load torque & variable $\mathrm{N} \cdot \mathrm{m}$ \\
\hline$W_{m \text { ref }}:$ reference speed & 200 and $300 \mathrm{rad} / \mathrm{s}$ \\
\hline$F_{c}$ : switching frequency & $4 \mathrm{kHz}$ \\
\hline$F_{s}:$ sampling frequency & $4 \mathrm{kHz}$ \\
\hline $1 T_{p}:$ unit time delay & $250 \mu \mathrm{s}$ \\
\hline$W_{m}:$ motor speed & 28 bits resolution \\
\hline$i_{a}:$ motor current & 12 bits resolution \\
\hline$d:$ duty cycle & 10 bits resolution \\
\hline Zero-order hold: fixed step discrete time & $250 \mu \mathrm{s}$ \\
\hline \multicolumn{2}{|l|}{$I_{3}:$ unity matrix of dimension 3} \\
\hline \multicolumn{2}{|l|}{$I_{7}:$ unity matrix of dimension 7} \\
\hline$\Gamma_{1}:$ updating gain & $0.0003 I_{3}$ \\
\hline$\Gamma_{2}:$ updating gain & $0.0003 I_{7}$ \\
\hline$C_{b e}:$ user-defined constant & 5 \\
\hline$c_{1}$ and $c_{2}:$ user-defined gains & 1 \\
\hline$c_{a}$ and $c_{c}:$ user-defined gains & 2.5 \\
\hline $\begin{array}{l}u_{a}: \text { initial control action defined by the } \\
\text { user }\end{array}$ & $30 \mathrm{~V}$ \\
\hline
\end{tabular}

It can be noticed from Remark 7 that low values of $C_{b e}$ lead to high control effort. For this reason, a small error region (low value of $C_{b e}$ ) causes saturation and faster response of the actuator. In case that the actuator cannot respond quickly, the condition of error region is not satisfied and the control design is not completely successful. In this way, the definition of $C_{b e}$ is a compromise between requirements of the output and actuator performance.

\section{Conclusions}

In all simulations, output error converges to a residual set defined by the user when the designed controller in this paper is applied to the plant. Small differences between experiment and theoretic results (Figures 4 and 5) are mainly due to hardware considerations and aspects related to the implementation which was not taken into account in the controller design. Some of them are delay in the control action, quantization effects which do not guarantee continuous control signal, noise and delays in the measured variables, 


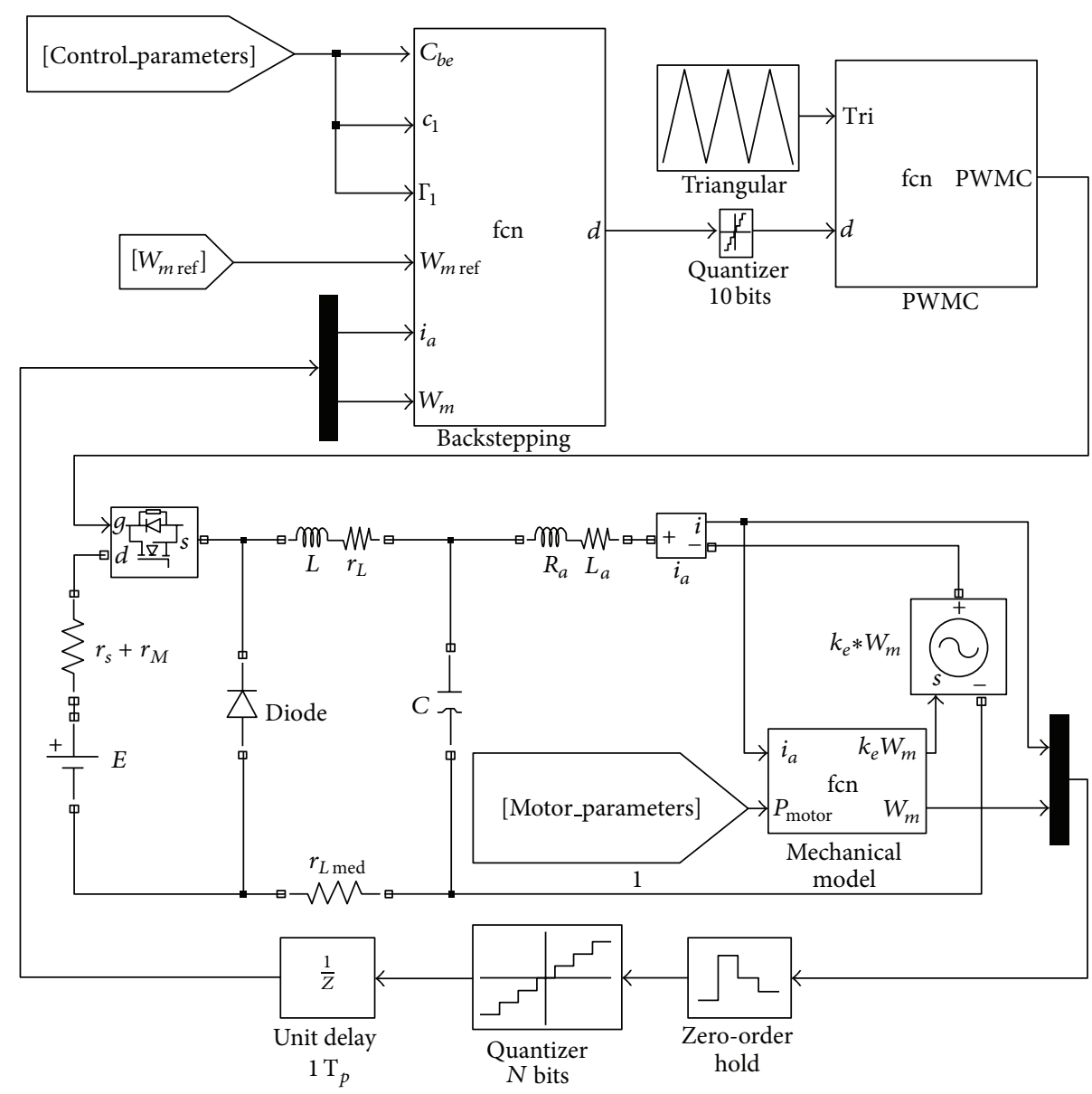

FIGURE 2: Sketch of the Simulink benchmark for simulation of the controller and motor system.

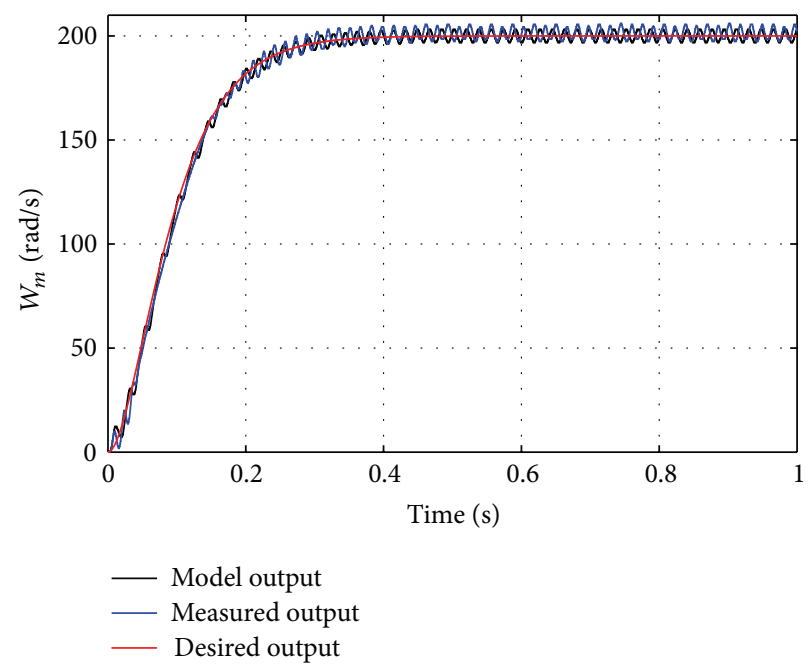

FIGURE 3: Simulated and experimental controller performance when $W_{m \text { ref }}=200 \mathrm{rad} / \mathrm{s}$.

and inaccuracy in the sensors. Nevertheless, starting from a complete unknown model, experiments and simulations show a high agreement, and experimental results validate the control technique, even in the cases when the set point is changed $50 \%$ of its initial value, and in the case when the load is changed $37 \%$ of its initial value. The designer must take into account the differences between experiments and simulations, prior to defining the error region.

The controller design based on the state adaptive backstepping involves a state transformation that provides two 


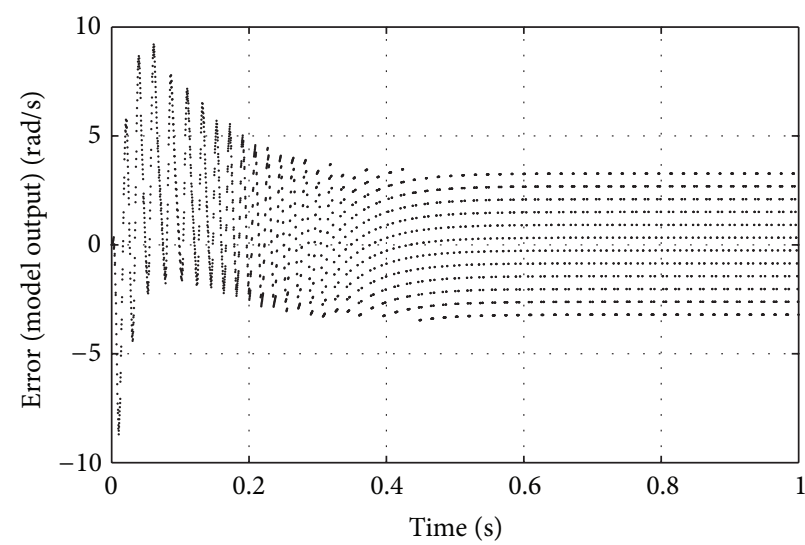

(a)

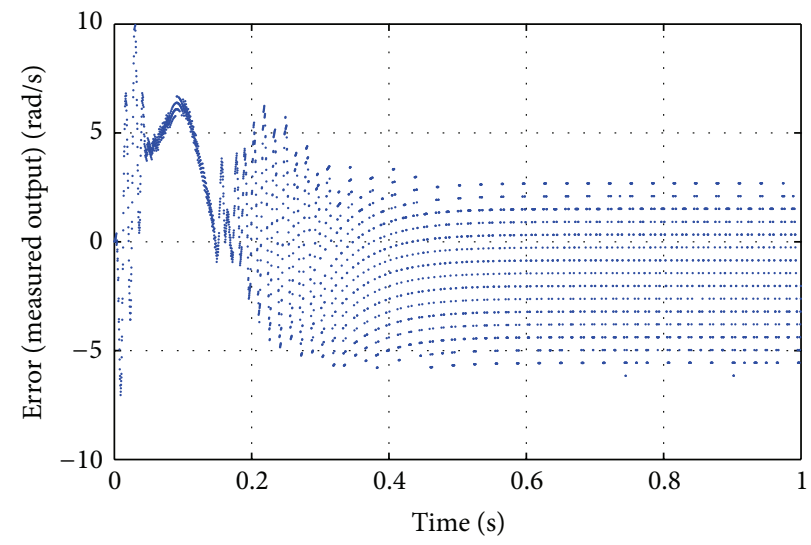

(b)

FIGURE 4: (a) Simulated and (b) experimental results for the tracking error when $W_{m \mathrm{ref}}=200 \mathrm{rad} / \mathrm{s}$.

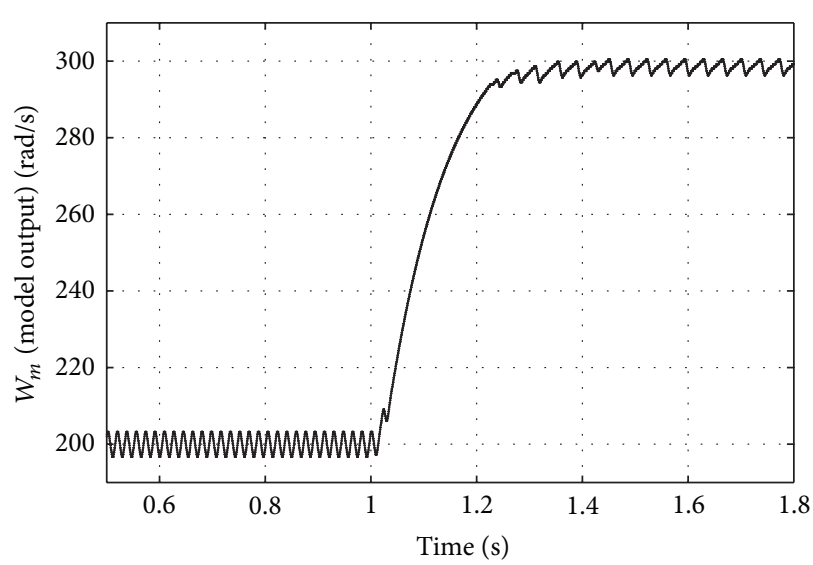

(a)

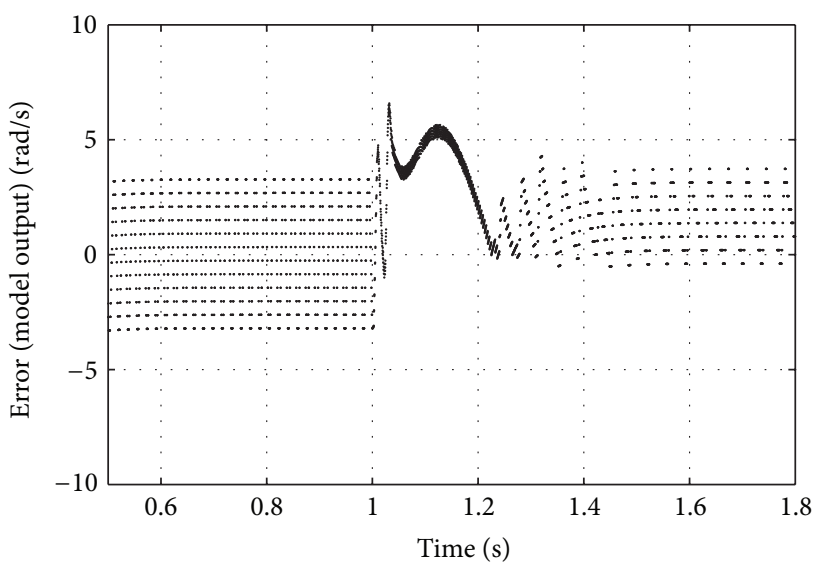

(c)

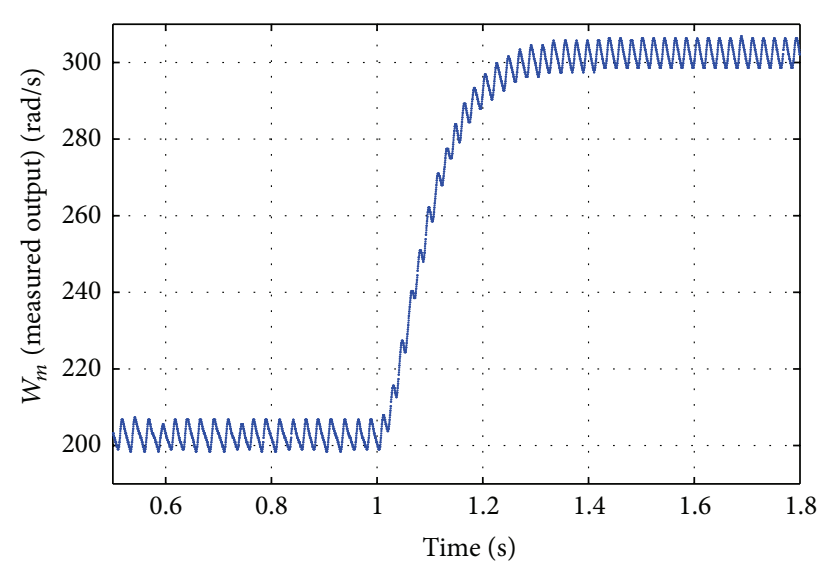

(b)

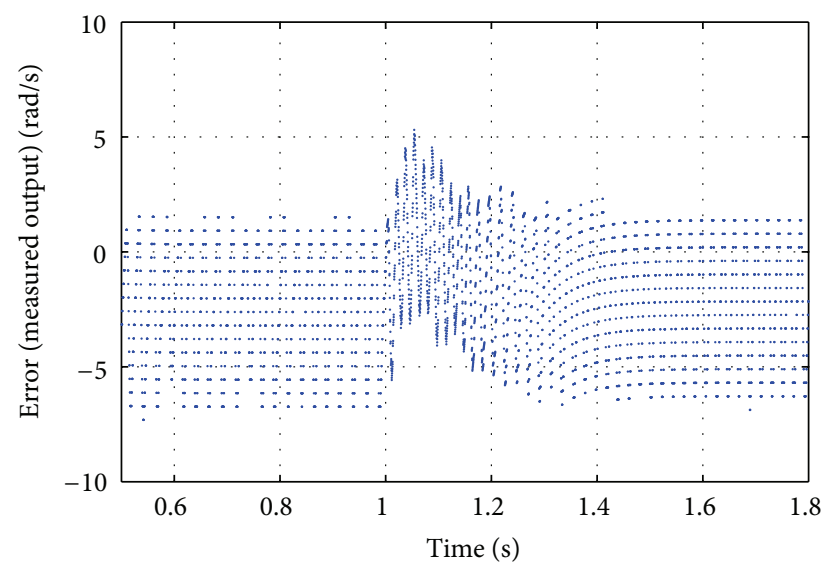

(d)

Figure 5: Simulated and experimental results when $W_{m \text { ref }}$ changes from $200 \mathrm{rad} / \mathrm{s}$ to $300 \mathrm{rad} / \mathrm{s}$ at $t=1 \mathrm{~s}$. (a), (c) simulated results. (b), (d) experimental results. 


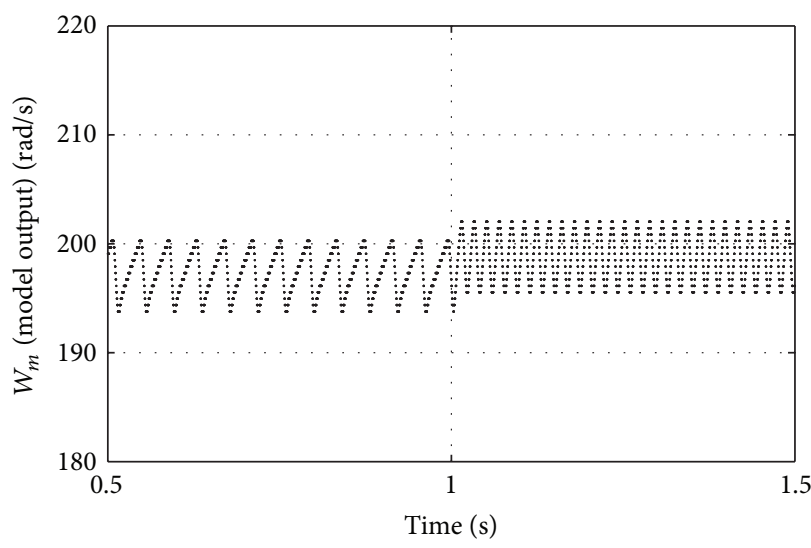

(a)

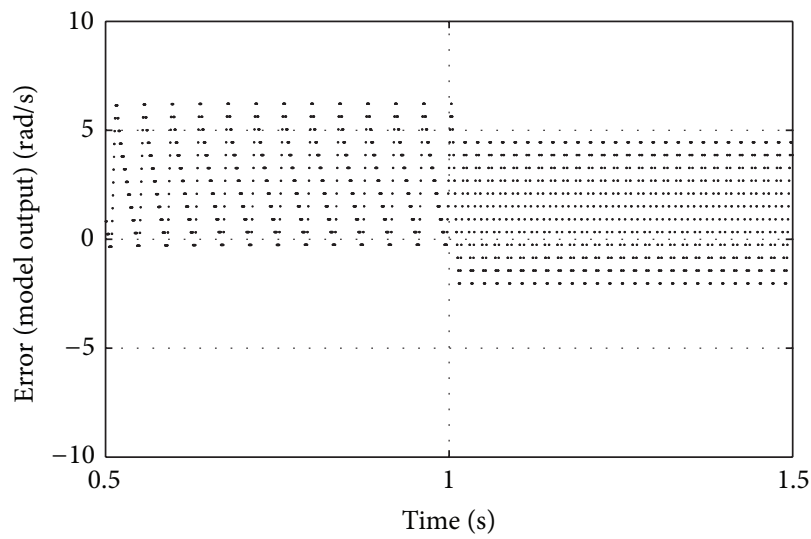

(c)

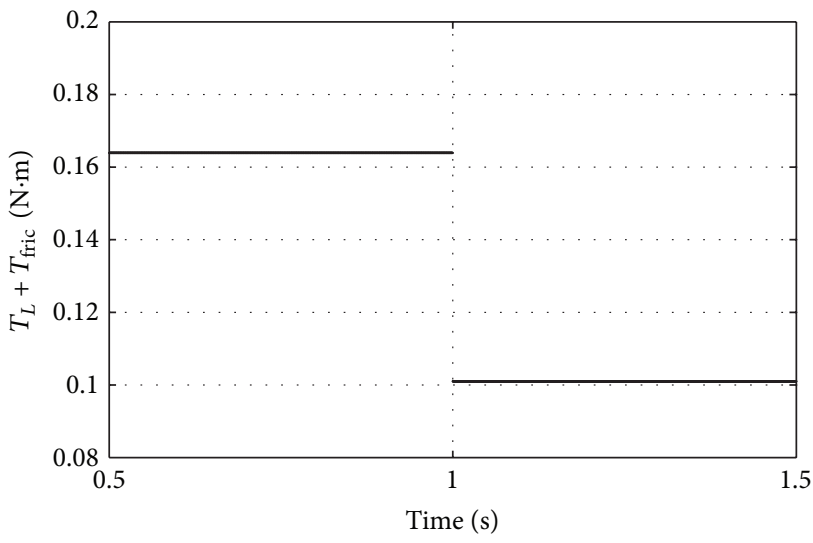

(e)

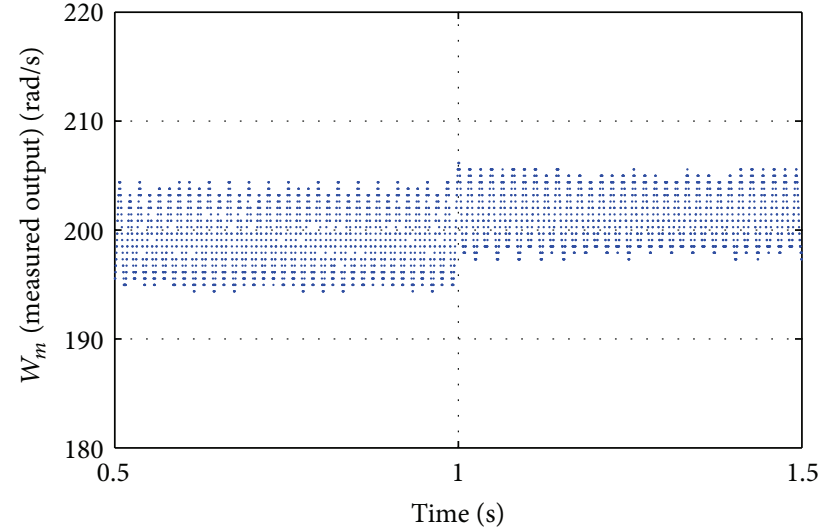

(b)

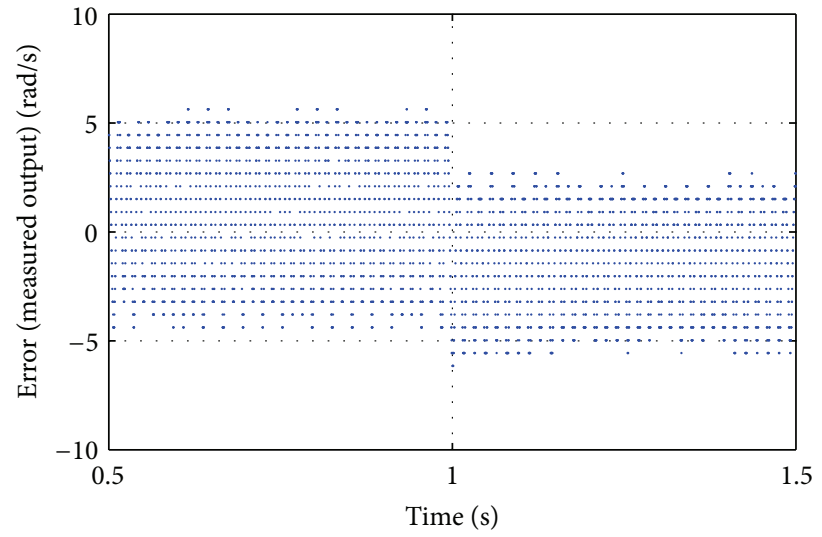

(d)

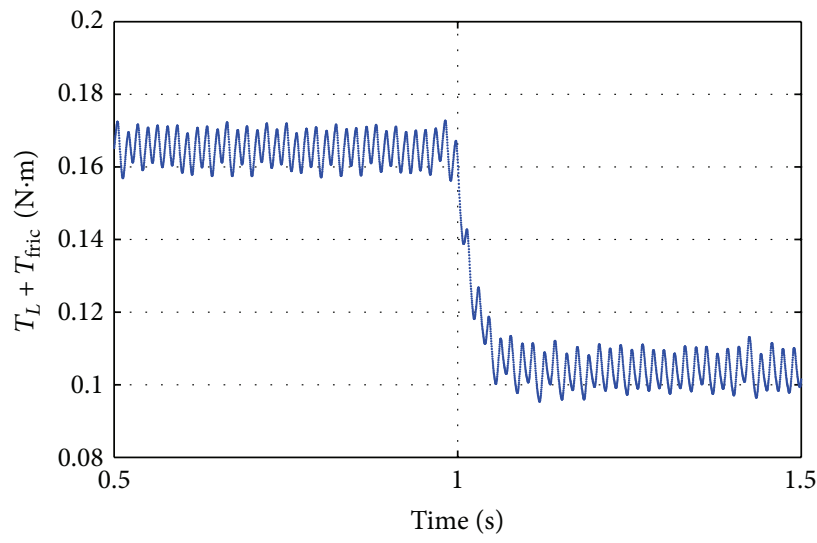

(f)

Figure 6: Simulated and experimental results when the load torque $T_{L}+T_{\text {fric }}$ changes from $0.1639 \mathrm{~N} \cdot \mathrm{m}$ to $0.101 \mathrm{~N} \cdot \mathrm{m}$ at $t=1 \mathrm{~s}$. (a), (c), (e) simulated results. (b), (d), (f) experimental results.

new states. The main elements to handle the unknown varying behavior of moment of inertia and load torque are introducing the upper bound of the model coefficients and introducing the lower bound of model coefficient $a_{2}$ in the parameterization.

With the aim to apply Lyapunov theory to demonstrate the stability of the controlled system a truncated quadratic function was formulated (Lyapunov-like function), in such a way that its magnitude and time derivative vanish when the new states reach a target region, which implied adequate properties of its time derivative.

The controller design and proof of boundedness and convergence properties are simpler in comparison to current works that use the Nussbaum gain technique. 


\section{Acknowledgments}

This work was partially supported by Universidad Nacional de Colombia-Manizales, Project 12475, Vicerrectoría de Investigación, DIMA, Resolution no. VR-2185.

\section{References}

[1] Z. Li, J. Chen, G. Zhang, and M. G. Gan, "Adaptive robust control for DC motors with input saturation," IET Control Theory \& Applications, vol. 5, no. 16, pp. 1895-1905, 2011.

[2] J. Linares-Flores, J. Reger, and H. Sira-Ramirez, "Load torque estimation and passivity-based control of a boostconverter/DC-motor combination," IEEE Transactions on Control Systems Technology, vol. 18, no. 6, pp. 1398-1405, 2010.

[3] M. A. Khanesar, O. Kaynak, and M. Teshnehlab, "Direct model reference Takagi-Sugeno fuzzy control of SISO nonlinear systems," IEEE Transactions on Fuzzy Systems, vol. 19, no. 5, pp. 914-924, 2011.

[4] I. Kanellakopoulos, P. V. Kokotović, and A. S. Morse, "Systematic design of adaptive controllers for feedback linearizable systems," IEEE Transactions on Automatic Control, vol. 36, no. 11, pp. 1241-1253, 1991.

[5] Y. Hong and B. Yao, "A globally stable saturated desired compensation adaptive robust control for linear motor systems with comparative experiments," Automatica, vol. 43, no. 10, pp. 1840-1848, 2007.

[6] S. Tong and Y. Li, "Fuzzy adaptive robust backstepping stabilization for SISO nonlinear systems with unknown virtual control direction," Information Sciences, vol. 180, no. 23, pp. 4619-4640, 2010.

[7] F. J. Lin, P. H. Shieh, and P. H. Chou, "Robust adaptive backstepping motion control of linear ultrasonic motors using fuzzy neural network," IEEE Transactions on Fuzzy Systems, vol. 16, no. 3, pp. 676-692, 2008.

[8] F. J. Lin, L. T. Teng, C. Y. Chen, and Y. C. Hung, "FPGA-based adaptive backstepping control system using RBFN for linear induction motor drive," IET Electric Power Applications, vol. 2, no. 6, pp. 325-340, 2008.

[9] A. El Magri, F. Giri, A. Abouloifa, and F. Z. Chaoui, "Robust control of synchronous motor through AC/DC/AC converters," Control Engineering Practice, vol. 18, no. 5, pp. 540-553, 2010.

[10] J. Zhou, C. Wen, and Y. Zhang, "Adaptive backstepping control of a class of uncertain nonlinear systems with unknown backlash-like hysteresis," IEEE Transactions on Automatic Control, vol. 49, no. 10, pp. 1751-1757, 2004.

[11] J. Zhou, C. Wen, and Y. Zhang, "Adaptive backstepping control of a class of uncertain nonlinear systems with unknown deadzone," in Proceedings of the IEEE Conference on Robotics, Automation and Mechatronics, pp. 513-518, December 2004.

[12] J. Zhou, C. Wen, and W. Wang, "Adaptive backstepping control of uncertain systems with unknown input time-delay," Automatica, vol. 45, no. 6, pp. 1415-1422, 2009.

[13] C. Wen, J. Zhou, and W. Wang, "Decentralized adaptive backstepping stabilization of interconnected systems with dynamic input and output interactions," Automatica, vol. 45, no. 1, pp. 55-67, 2009.

[14] A.-C. Huang and Y.-S. Kuo, "Sliding control of non-linear systems containing time-varying uncertainties with unknown bounds," International Journal of Control, vol. 74, no. 3, pp. 252$264,2001$.
[15] Y. Zhang, P. Y. Peng, and Z. P. Jiang, "Stable neural controller design for unknown nonlinear systems using backstepping," IEEE Transactions on Neural Networks, vol. 11, no. 6, pp. 13471360,2000

[16] C. P. Bechlioulis and G. A. Rovithakis, "Adaptive control with guaranteed transient and steady state tracking error bounds for strict feedback systems," Automatica, vol. 45, no. 2, pp. 532-538, 2009.

[17] Y. Li, S. Qiang, X. Zhuang, and O. Kaynak, "Robust and adaptive backstepping control for nonlinear systems using RBF neural networks," IEEE Transactions on Neural Networks, vol. 15, no. 3, pp. 693-701, 2004.

[18] J. Na, X. Ren, G. Herrmann, and Z. Qiao, "Adaptive neural dynamic surface control for servo systems with unknown deadzone," Control Engineering Practice, vol. 19, no. 11, pp. 1328-1343, 2011.

[19] D. Wang and J. Huang, "Neural network-based adaptive dynamic surface control for a class of uncertain nonlinear systems in strict-feedback form," IEEE Transactions on Neural Networks, vol. 16, no. 1, pp. 195-202, 2005.

[20] C.-Y. Su, Y. Feng, H. Hong, and X. Chen, "Adaptive control of system involving complex hysteretic nonlinearities: a generalised Prandtl-Ishlinskii modelling approach," International Journal of Control, vol. 82, no. 10, pp. 1786-1793, 2009.

[21] Y. Feng, H. Hong, X. Chen, and C. Y. Su, "Robust adaptive controller design for a class of nonlinear systems preceded by generalized Prandtl-Ishlinskii hysteresis representation," in Proceedings of the 7th World Congress on Intelligent Control and Automation (WCICA '08), pp. 382-387, Chongqing, China, June 2008.

[22] Y. Feng, C. Y. Su, and H. Hong, "Universal construction of robust adaptive control laws for a class of nonlinear systems preceded by generalized Prandtl-Ishlinskii representation," in Proceedings of the 3rd IEEE Conference on Industrial Electronics and Applications (ICIEA '08), pp. 153-158, Singapore, June 2008.

[23] Y. Feng, Y. M. Hu, and C. Y. Su, "Robust adaptive control for a class of perturbed strict-feedback nonlinear systems with unknown Prandtl-Ishlinskii hysteresis," in Proceedings of the IEEE International Symposium on Intelligent Control (ISIC '06), pp. 106-111, Munich, Germany, October 2006.

[24] H. Du, S. S. Ge, and J. K. Liu, "Adaptive neural network output feedback control for a class of non-affine non-linear systems with unmodelled dynamics," IET Control Theory and Applications, vol. 5, no. 3, pp. 465-477, 2011.

[25] S. S. Ge and J. Wang, "Robust adaptive tracking for timevarying uncertain nonlinear systems with unknown control coefficients," IEEE Transactions on Automatic Control, vol. 48, no. 8, pp. 1463-1469, 2003.

[26] Y. Xudong and J. Jingping, "Adaptive nonlinear design without a priori knowledge of control directions," IEEE Transactions on Automatic Control, vol. 43, no. 11, pp. 1617-1621, 1998.

[27] S. S. Ge and J. Wang, "Robust adaptive neural control for a class of perturbed strict feedback nonlinear systems," IEEE Transactions on Neural Networks, vol. 13, no. 6, pp. 1409-1419, 2002.

[28] R. D. Nussbaum, "Some remarks on a conjecture in parameter adaptive control," Systems and Control Letters, vol. 3, no. 5, pp. 243-246, 1983.

[29] K. Astrom and B. Wittenmark, Adaptive Control, AddisonWesly, 2nd edition, 1995.

[30] S. Tong, C. Liu, and Y. Li, "Fuzzy-adaptive decentralized output-feedback control for large-scale nonlinear systems with 
dynamical uncertainties," IEEE Transactions on Fuzzy Systems, vol. 18, no. 5, pp. 845-861, 2010.

[31] H. E. Psillakis, "Further results on the use of nussbaum gains in adaptive neural network control," IEEE Transactions on Automatic Control, vol. 55, no. 12, pp. 2841-2846, 2010.

[32] A. Rincon, F. Angulo, and G. Osorio, "A robust state feedback adaptive controller with improved transient tracking error bounds for plants with unknown varying control gain," in Applications of Nonlinear Control, chapter 5, pp. 79-98, INTECH, 2012.

[33] M. M. Polycarpou and P. A. Ioannou, "On the existence and uniqueness of solutions in adaptive control systems," IEEE Transactions on Automatic Control, vol. 38, no. 3, pp. 474-479, 1993.

[34] J. Slotine and W. Li, Applied Nonlinear Control, Prentice Hall, Englewood Cliffs, NJ, USA, 1991.

[35] A. Leva, L. Piroddi, M. Di Felice, A. Boer, and R. Paganini, "Adaptive relay-based control of household freezers with on-off actuators," Control Engineering Practice, vol. 18, no. 1, pp. 94-102, 2010.

[36] M. D. Felice, L. Piroddi, A. Leva, and A. Boer, "Adaptive temperature control of a household refrigerator," in Proceedings of the American Control Conference (ACC '09), pp. 889-894, St. Louis, Mo, USA, June 2009.

[37] H. Royden, Real Analysis, Prentice Hall, Upper Saddle River, NJ, USA, 1988.

[38] P. Ioannou and J. Sun, Robust Adaptive Control, Prentice Hall, Upper Saddle River, NJ, USA, 1996. 


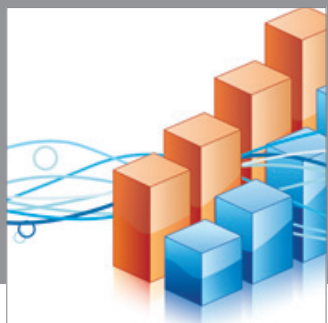

Advances in

Operations Research

mansans

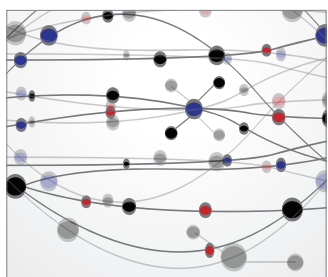

The Scientific World Journal
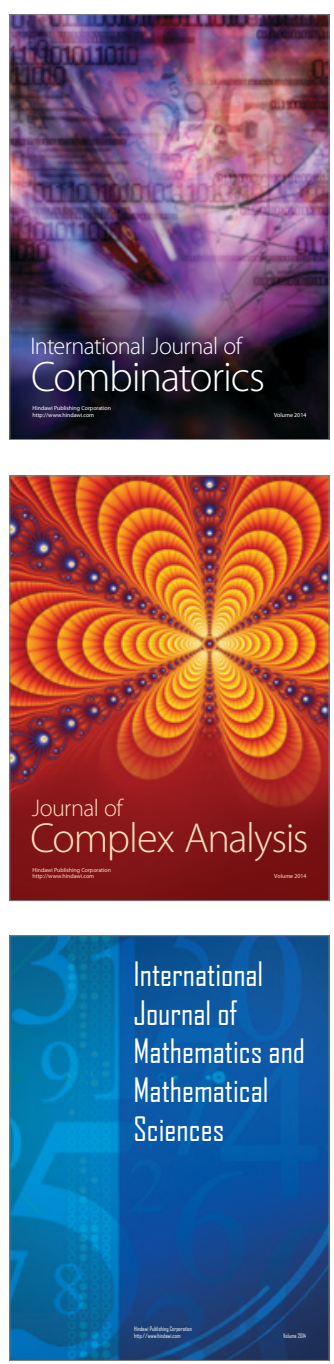
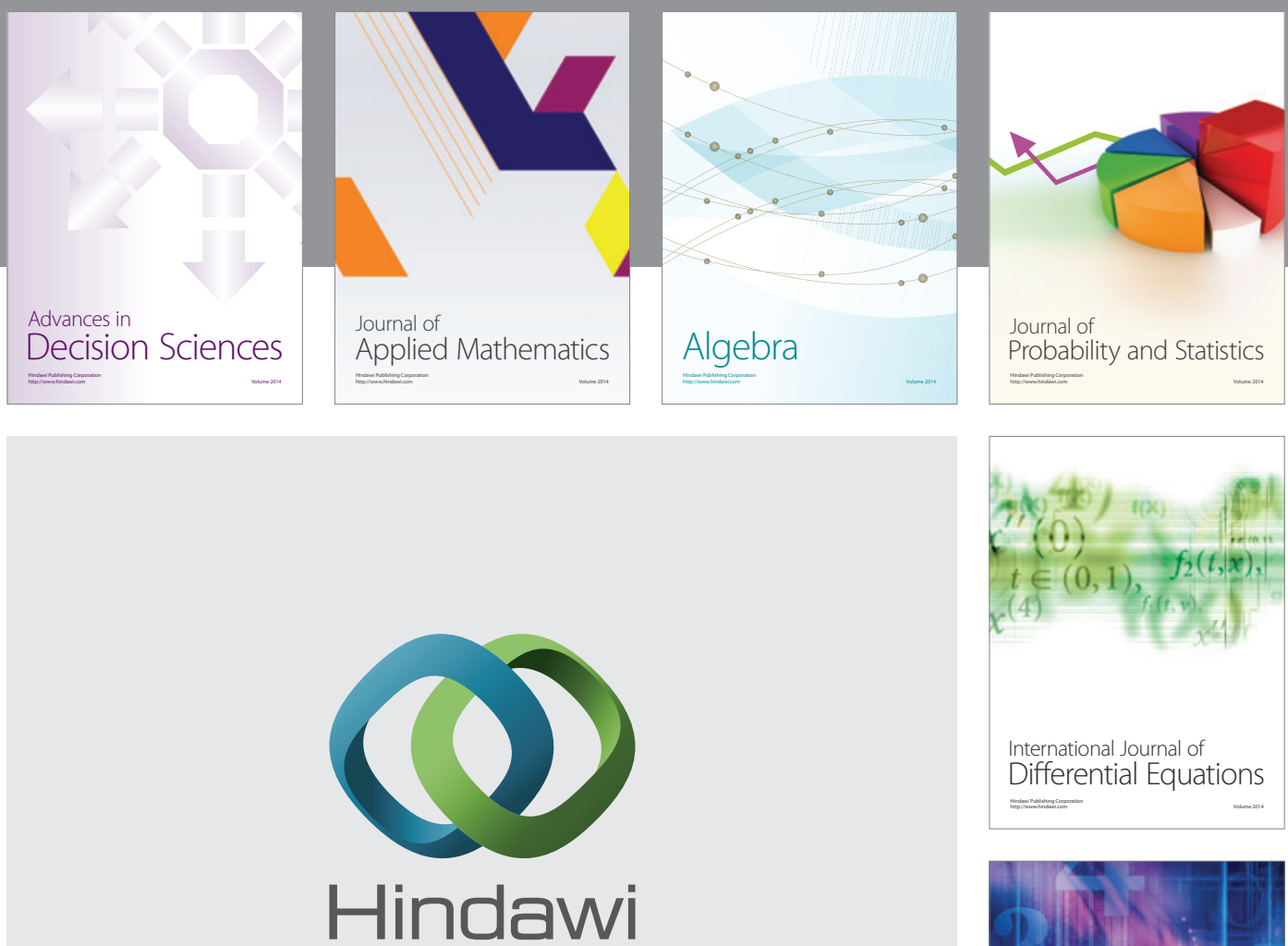

Submit your manuscripts at http://www.hindawi.com
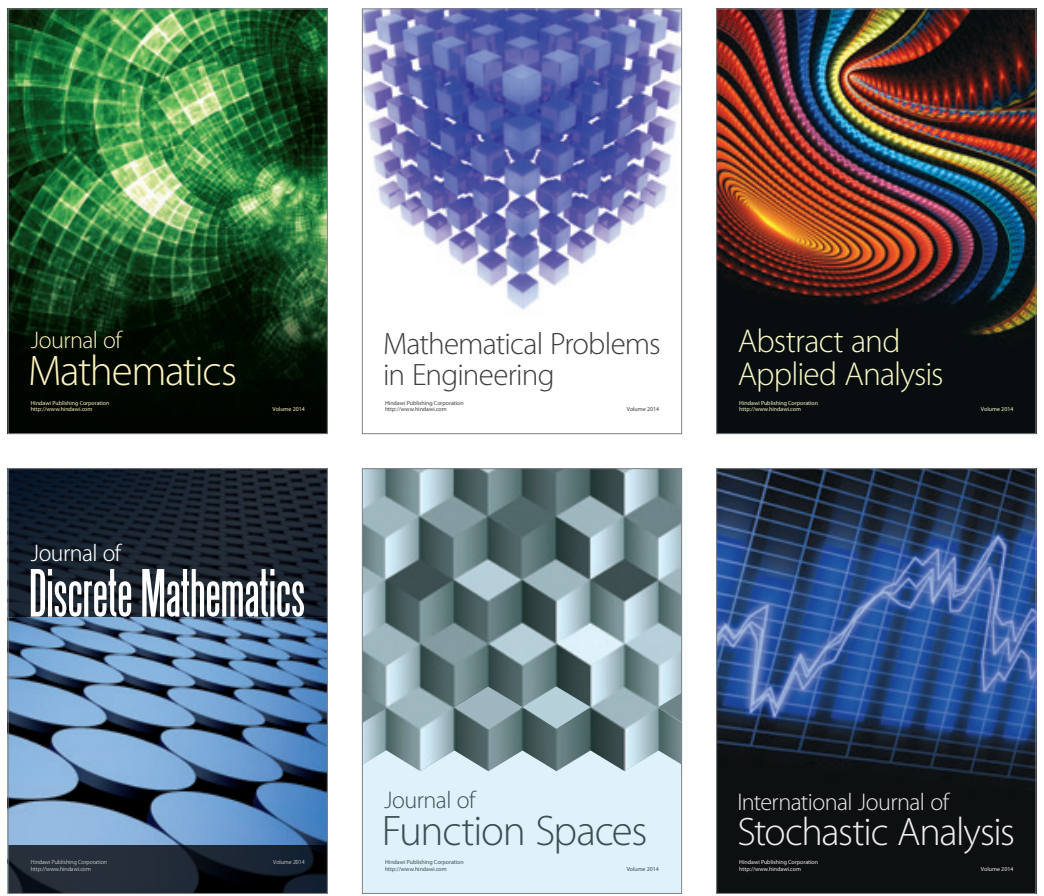

Journal of

Function Spaces

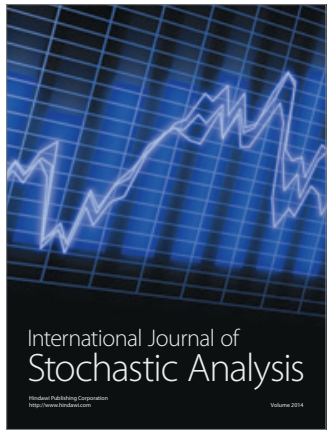

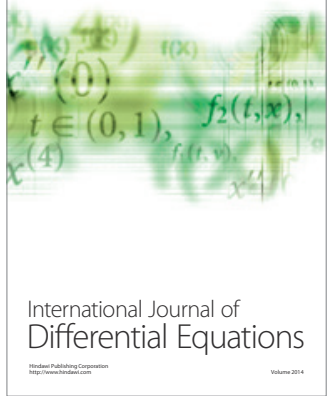
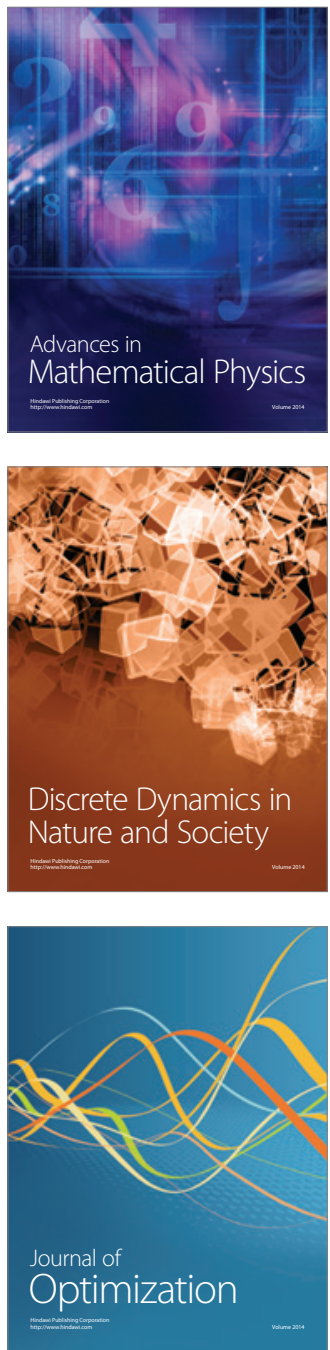